\title{
Acknowledgement to Reviewers of Energies in 2018
}

\author{
Energies Editorial Office
}

MDPI, St. Alban-Anlage 66, 4052 Basel, Switzerland

Published: 8 January 2019

Rigorous peer-review is the corner-stone of high-quality academic publishing. The editorial team greatly appreciates the reviewers who contributed their knowledge and expertise to the journal's editorial process over the past 12 months. In 2018, a total of 3588 papers were published in the journal, with a median time to first decision of 14.39 days and a median time to publication of 35 days. The editors would like to express their sincere gratitude to the following reviewers for their cooperation and dedication in 2018:

\begin{tabular}{|c|c|}
\hline A. M. Araújo, Rui & Ahmadieh Khanesar, Mojtab \\
\hline Abbas Jibrin, Abubakar & Ahn, Byung Tae \\
\hline Abbas, Azhar & Ahn, Dukju \\
\hline Abbaszadeh, Shiva & Ahn, Hyung Keun \\
\hline Abdallah, Shaaban & Ahvazi, Behzad \\
\hline Abdel-Motaleb, Ibrahim M. & Akander, Jan \\
\hline Abdel-Salam, Mazen & Akanji, Lateef \\
\hline Abdo, Hafez & Akih-Kumgeh, Ben \\
\hline Abdollahi, Amir & Akitsu, Tetsuya \\
\hline Abdollahzadeh Jamalabadi, Mohammad & Akkutlu, I. Yucel \\
\hline Yaghoub & Aksanli, Baris \\
\hline Abedi, Sara & Al Ameri, Ahmed \\
\hline Abesser, Corinna & Al Dushaishi, Mohammed \\
\hline Ábrego, Javier & Ala, Guido \\
\hline Abreu, Antonio & Al-Abadi, Ali \\
\hline Abualfaraj, Noura & Alam, S M Shafiul \\
\hline Abu-Siada, Ahmed & Alamaniotis, Miltiadis \\
\hline Abuzneid, Abdel-Shakour & Alander, Jarmo \\
\hline Accardo, Grazia & Al-Anssari, Sarmad \\
\hline Aceves, Salvador & Alarco, Jose \\
\hline Achinivu, Ezinne & Albano, Maurizio \\
\hline Adamo, Alessandro D' & Albarracín, Ricardo \\
\hline Adamovský, Radomír & Alberello, Alberto \\
\hline Adebisi, Bamidele & Albergante, Luca \\
\hline Adekunle, Timothy O. & Alberto, Perea Moreno \\
\hline Adiletta, Giuseppina & Albizu, Igor \\
\hline Aditjandra, Paulus Teguh & Albu, Mihaela \\
\hline Adriaens, Peter & Alcarria, Ramon \\
\hline Afgan, Imran & Alcayde García, Alfredo \\
\hline Afionis, Stavros & Alcázar-Ortega, Manuel \\
\hline Afonso, Armando Silva & Alconero, Patricia Luis \\
\hline Afonso, Clito & Aldazabal, Javier \\
\hline Afonso, Frederico & Aldieri, Luigi \\
\hline Afzal, Muhammad & Ale-Emran, Sayyed Mahdi \\
\hline Agafonov, Vadim & Aleluia Da Silva Reis, Lara \\
\hline Agnew, Brian & Aleshin, Sergey \\
\hline Agonafir, Mesfin Belayneh & Alessandra, Romolo \\
\hline Ahmad, Muhammad Waseem & Alessandro, Di Giorgio \\
\hline Ahmadalipour, Ali & Alessandro, Sbrana \\
\hline Ahmadi, Mohammad Hosein & Alexandridis, Antonio \\
\hline
\end{tabular}


Alfano, Vincenzo

Alfieri, Luisa

Algarín, Carlos Robles

Ali Nasir, Muhammad

Ali, Aamer

Ali, Babkir

Ali, Mohamed Abdelmonem

Aliabadi, Amir A.

Alin, Bosioc

Alizad, Karim

Aljankawey, Abdualah S.

Allard, Bruno

Allmaier, Hannes

Alluri, Nagamalleswara Rao

Ally, Moonis Raza

Almeida Dávi, Giovani

Almeida, Ricardo

Al-Mudhafar, Watheq J.

Almutairi, Abdulrahman Soud

Alobaid, Falah

Alsharif, Mohammed

Altan, Hasim

Altin, Müfit

Álvarez Antón, Juan Carlos

Álvarez Fernández, Martina Inmaculada

Álvarez Pardiñas, Angel

Álvarez, Fernando

Alvarez-Diaz, Marcos

Alvisi, Stefano

Amado, Miguel

Amasawa, Eri

Amezcua, Javier

Amin, Mohammad

Amin, Sakib-Bin

Amini, Hadi

Amirat, Yassine

Amiryar, Mustafa E.

Amosu, Adewale

Amuakwa-Mensah, Franklin

Amutio Izaguirre, Maider

An, Chunjiang

Anand, Vijay

Ancellin, Matthieu

Ancona, Maria Alessandra

Andersen, Pal Ostebo

Andersen, Søren J.

Anderson, Kevin R.

Anderson, Ryan

Andoga, Rudolf

Andreassi, Luca

Andreescu, Gheorghe-Daniel

Andreoli, Enrico

Andreopoulou, Zacharoula

Andritsch, Thomas

Andriukaitis, Darius

Anghel, Ionut

Angrecka, Sabina

Angus, Doug
Anselmet, Fabien

Anta, Adolfo

Antchev, Mihail Hristov

Anthony, Ben

Antonopoulos, Angelos

Anttonen, Markku

Antuchevičienè, Jurgita

Antuñano, Aitor Ciarreta

Aoki, Mutsumi

Apergis, Nicholas

Aprea, Ciro

Aprile, Marcello

Apsley, Judith M.

Arabatzis, Garyfallos

Arahal, Manuel R.

Aramendia, Inigo

Araneo, Rodolfo

Araoz, Joseph A.

Arato, Elisabetta

Arcos-Vargas, Angel

Arcuri, Natale

Ardila-Rey, Jorge Alfredo

Aretakis, Nikolaos

Ariga, Katsuhiko

Arima, Hirofumi

Arina, Renzo

Ariyur, Kartik

Arkar, Ciril

Armadillo, Egidio

Arnold, Daniel

Arora, Sameer

Arrabe, Ricardo Granizo

Arroyo, Oscar

Artal-Sevil, Jesús Sergio

Arteconi, Alessia

Artiukhina, Ekaterina

Arvanitis, Konstantinos

Arwatz, Gilad

Aryal, Nabin

Aryana, Saman

Asif, Amir

Asorey-Cacheda, Rafael

Astarloa, Armando

Astiaso Garcia, Davide

Asuka, Jusen

Atanasoae, Pavel

Atazadeh, Behnam

Athanasiou, Christos

Attya, Ayman

Atukeren, Erdal

Auffermann, Gudrun

Aula, Fadhil T.

Aung, Kendrick T.

Ausloos, Marcel

Avenas, Yvan

Avendaño-Valencia, Luis David

Averbukh, Moshe

Avery, James 
Avgouropoulos, George

Avulapati, Madan Mohan

Awerbuch, Jonathan

Aye, Lu

Ayele, Yonas Zewdu

Azbe, Valentin

Aziz, Muhammad

Azizi, Mohsen

Azman, Samet

Ba, Chien Nguyen

Babacan, Oytun

Babaei, Mahnoush

Babazadeh, Davood

Babiarz, Bożena

Bach, Christian

Bachaga, Tarek

Baczyński, Dariusz

Badcock, Rod

Badihi, Hamed

Badulescu, Alina

Badyda, Krzysztof

Bae, Woorham

Bae, Youngchul

Baeyens, Jan

Bagheri, Mehdi

Bahar, Mohammad

Bahmani, Hamed

Bahmani, Sahar

Bahrami, Shahab

Bai, Attila

Bai, Kevin (Hua)

Bai, Xianglan

Baig, Hasan

Baimel, Dmitry

Bajracharya, Arun

Baker, Kyri A.

Baker, Thar

Bakhtiyarov, Sayavur

Bakken Sperstad, Iver

Balacco, Gabriella

Balaguer, Jacint

Balasingam, Suresh Kannan

Balázs, Horváth

Baldi, Simone

Baldini, Mario

Baležentis, Tomas

Bálint, Erika

Ballottari, Matteo

Balocco, Carla

Balomenos, Efthimios

Banaszak, Szymon

Banchero, Mauro

Bandi, Chaithanya

Banerjee, Aparajita

Bangga, Galih

Banguero, Edison

Bañón, Luis

Baños Navarro, Raúl
Bányai, Tamás

Bao, Shaowu

Bao, Yi

Baptista, José

Barambones, Oscar

Baranwal, Rohit

Baranyai, Nóra Hegedűsné

Barata, Filipe

Barata, Jorge M M

Barati, Masoud

Barbanti, Lorenzo

Barbato, Marco

Barbato, Maurizio

Barbieri, Jacopo

Barbosa, Ramiro S.

Barbu, Marian

Bardweel, Hamzeh

Barez, Fred

Barfidokht, Abbas

Bargallo, Ramon

Bar-Kohany, Tali

Barlas, Athanasios

Barmada, Sami

Barnes, Frank S.

Barone, Giuseppe

Barote, Luminita

Barré, Olivier

Barreca, Francesco

Barreras, Jorge Varela

Barrero-González, Fermín

Barrios Muriel, Jorge

Bartlomiejczyk, Mikolaj

Bartocci, Pietro

Barton, John

Bartoňová, Lucie

Bascompta, Marc

Baser, Tugce

Bastante-Ceca, Maria Jose

Bastiaans, Rob

Basu, Malabika

Batard, Christophe

Battista, Federico

Battistel, Alberto

Battisti, Alessandra

Batzelis, Efstratios

Baudry, Gino

Baumann, Manuel

Bawaneh, Khaled

Bayer, Patrick

Bayraktar, Emin

Bayram, I. Safak

Bazydło, Grzegorz

Beach, Tom

Beardsmore, Graeme

Beath, Andrew

Beck, Yuval

Beckett, Christopher

Bedon, Chiara 
Beganovic, Nejra

Behrou, Reza

Beizaee, Arash

Belardini, Elisabetta

Beleza De Carvalho, José António

Belke, Ansgar H.

Bell, John

Bella, Federico

Bellardita, Marianna

Bellia, Laura

Bellido, Francisco J.

Bellos, Evangelos

Belmont, Mike

Belussi, Lorenzo

Benato, Alberto

Benato, Roberto

Benavente-Peces, Cesar

Bengtsson, Magnus

Benhmad, François

Beniak, Juraj

Benim, Ali

Benim, Ali Cemal

Bennadji, Hayat

Bennett, Terry

Bensmann, Astrid

Bentsen, Niclas Scott

Berardi, Umberto

Berg, Steffen

Bergillos, Rafael J.

Bernardino, Mariana

Bertoldo, Silvano

Bertrand, Martine

Besagni, Giorgio

Besharat, Mohsen

Besio, Giovanni

Bessa, Ricardo

Beyerlein, Steven

Bhandari, Khagendra

Bhandari, Ramchandra

Bhatarai, Bishnu

Bhattacharjya, Dhrubajyoti

Bhattacharya, Subhashish

Bianchi, Giuseppe

Bianco, Vincenzo

Bidram, Ali

Bielaczyc, Piotr

Bien, Andrzej

Bignucolo, Fabio

Bilan, Yuriy

Bilas, Vedran

Bilbao, Julia

Biniak-Pieróg, Małgorzata

Birdwell, Justin

Biserni, Cesare

Bishop, Justin

Biswas, Kaushik

Biswas, Soumyajyoti

Bithas, Kostas
Bithas, Petros S.

Bizon, Nicu

Blakers, Andrew

Blanchard, Richard

Blanco, Juan A.

Blanco, Pilar

Blandin, Gaetan

Blazejcyk-Majka, Lucyna

Blazevic, Zoran

Blecich, Paolo

Bletterie, Benoît

Block, Kornelis

Blokhus, Anne Marit

Boada, Beatriz L.

Boada, Maria Jesus Lopez

Boait, Peter

Boak, Jeremy

Boek, Edo

Bohling, Björn

Bohutskyi, Pavlo

Boldea, Ion

Bollen, Math

Bölöni, Ladislau

Bolotov, Leonid

Bolzmacher, Christian

Bom, Pedro Duarte

Bonelli, Francesco

Bonfiglio, Andrea

Borge-Diez, David

Borges, Cruz E.

Borkowski, Piotr

Bornatico, Raffaele

Borochkin, Alexander

Borole, Abhijeet P.

Borowski, Marek

Borozan, Djula

Borquist, Eric

Borrego, Carlos

Borz, Stelian Alexandru

Boschetti, Tiziano

Bosich, Daniele

Boškoski, Pavle

Bosma, Bret

Bossanyi, Ervin

Bosso, Luciano

Bostan, Ionel

Bottaccioli, Lorenzo

Bottacin Busolin, Andrea

Bottiglione, Francesco

Boudissa, Rabah

Bougdah, Hocine

Bouhouras, Aggelos S.

Boulahouache, Chaouki

Bourg, Stéphane

Bouris, Demetri

Bouwmans, Thierry

Bouzid, Allal

Bozalakov, Dimitar 
Bracale, Antonio

Braccio, Giacobbe

Brancato, Virginia

Brassai, Sándor Tihamér

Brecl, Kristijan

Breitsamter, Christian

Bremer, Jörg

Brent, Alan

Brereton, Patrick

Bretterklieber, Thomas

Briga-Sá, Ana

Brignone, Massimo

Brillard, Alain

Brinkerhoff, Joshua

Briois, Pascal

Brito, Miguel Centeno

Brito, Moisés

Brockway, Paul

Brójo, Francisco

Bronshtein, Svetlana

Browell, Jethro

Brown, David

Brown, Mark

Brown, Robert Keith

Brown, Tom

Bruckman, Viktor

Brueckner, Markus

Bruland, Oddbjørn

Brun, Nicolas

Brunerová, Anna

Brunner, Andreas

Bruno, Laura

Brunone, Bruno

Bruzzone, Luca

Brzeski, Piotr

$\mathrm{Bu}$, Siqi

Buccella, Concettina

Buccino, Mariano

Buckman, Jim

Buczyński, Rafał

Budhi, Sridhar

Bueno, Emilio

Bueno, Emilio J.

Bugajski, Piotr

Bui, Mai

Bui, Tinh Quoc

Bujnowski, Adam

Bukata, Bala Boyi

Buker, Mahmut Sami

Bulgariu, Laura

Bulic, Neven

Bullough, John

Bunker, Kaitlyn J.

Bunn, Derek

Bunster, Victor

Buratti, Cinzia

Burdett, Robert

Burluka, Alexey
Burnos, Piotr

Burugupally, Sindhu Preetham

Busby, Jonathan

Buscarino, Arturo

Bush, Brian W

Bustamante, Eliseo

Busu, Mihail

Buticchi, Giampaolo

Butusov, Denis

Byttner, Stefan

Caballero, Alvaro

Cabot, Joan Marc

Cabral-Pinto, Marina M. S.

Caetano, Nídia

Caggiano, Alessandra

Caggiano, Antonio

Cagnano, Alessia

Cai, Feiyang

Cai, Hongzhu

Cai, Hua

Cai, Jie

Caignaert, Guy

Calado, Maria Do Rosário

Caldas, Paulo

Calif, Rudy

Calin, Adrian Cantemir

Calisal, Sander

Calka, Andrzej

Calleja, Amaia

Callejón, Ángel

Calore, Enrico

Calzada, Igor

Camacho, Antonio

Cameron, Joseph

Cametti, Cesare

Campagnolo, Filippo

Campana, Pietro

Campbell, Carlene

Campo, Celeste

Campora, Ugo

Camporeale, Rosalia

Campos, Luis Braga

Cañas-Carretón, Miguel

Cancelliere, Michel

Canders, Wolf

Candolfi, Christophe

Candrea, Adina Nicoleta

Canepa, Edward

Cañete, Eduardo

Caniato, Marco

Cannavale, Alessandro

Cannistraro, Giuseppe

Cannistraro, Mauro

Cano Garcia, Jose Manuel

Cano, Antonio

Cansino, Jose M.

Cao, Toan

Capasso, Clemente 
Capata, Roberto

Capella, Juan Vicente

Capezzali, Massimiliano

Capizzi, Giacomo

Caporale, Andrea

Capsoni, Doretta

Carabano, Rocio

Carabineiro, Sónia

Caramanis, Michael

Carattini, Stefano

Carbajales-Dale, Michael

Carbone, Rosario

Carli, Raffaele

Carli, Stefano

Carlucci, Antonio Paolo

Carmona, Luis Gabriel

Carou, Diego

Carpinelli, Guido

Carpita, Mauro

Carravetta, Armando

Carreño-Ortega, Angel

Carrillo, Camilo

Carrión, Miguel

Caruntu, Constantin Florin

Carutasu, George

Cascone, Stefano

Cassetti, Gabriele

Castanheiro, José

Castellani, Beatrice

Castellani, Francesco

Castellanos, Sergio

Castelli-Dezza, Francesco

Castelló Moreno, Jaime

Casteren, Jan Van

Castiglione, Arcangelo

Castilla, Roberto

Castillo, Oscar

Castillo-Ruiz, Francisco J.

Castro, Eulogio

Catalano, Pasquale

Cataldo, Filippo

Catalin, Alexandru

Catalin, Petrescu

Cavallaro, Fausto

Cavallaro, Giuseppe

Cavallaro, Luca

Cazacu, Emil

Cecconet, Daniele

Cecilia, Juan Antonio

Cecílio, José

Celik, Berk

Cen, Yinjie

Cenek, Martin

Centobelli, Piera

Ceola, Serena

Cerchione, Roberto

Cernat, Mihai

Cerqueti, Roy
Cerro-Prada, Elena

Cervelló-Pastor, Cristina

Cetin, Kristen S.

Cha, Minsu

Chadli, Mohammed

Chai, Lei

Chai, Merlin

Chakraborty, Uday Kumar

Chalashkanov, Nikola

Chalvatzis, Konstantinos

Chamonine, Mikhail

Chan, Leon

Chand, Shyam

Chandrasekhar, Arunkumar

Chang, Chia-Lin

Chang, Daejun

Chang, En-Chih

Chang, Henry-S.M.

Chang, Hsueh-Hsien

Chang, Junghwan

Chang, Seongju

Chang, Tian-Pau

Chang, Wan-Jung

Chang, Wen-Yeau

Chang, Yau-Zen

Chang, Yu-Chung

Chao, Ru-Min

Chapman, Andrew John

Chapman, Jamie

Charfeddine, Lanouar

Charisiou, Nikolaos

Charvatova, Hana

Chatterjee, Sabornie

Chatzakis, John

Chatzipoulka, Christina

Chaudhury, Anwesha

Chava, Rama Krishna

Chaves Ávila, José Pablo

Chayaamor-Heil, Natasha

Che, Liang

Chehouri, Adam

Chen, Bo

Chen, Bo-Hao

Chen, Boli

Chen, Chi-Hua

Chen, Chiing-Chang

Chen, Chunhui

Chen, Chun-Ta

Chen, Fang

Chen, Genshe

Chen, Hailong

Chen, Jinhua

Chen, Jun

Chen, Kai-Sheng

Chen, Keng-yuan

Chen, Kuan

Chen, Kuo-Ching

Chen, Liang-Bi 
Chen, Liang-Yu

Chen, Lien-Wu

Chen, Lin

Chen, Lung-Chien

Chen, Ming-Hung

Chen, Paochi

Chen, Ruei-Tang

Chen, Shengen

Chen, Shengnan (Nancy)

Chen, Shin-Ju

Chen, Shuo

Chen, Tai-Cheng

Chen, Xiongyu

Chen, Yan

Chen, Yangkang

Chen, Yen-Hui

Chen, Yi-Chung

Chen, Yie-Tone

Chen, Yong-Song

Chen, Yuan-Tsung

Chen, Zhangxing John

Chen, Zhi

Chen, Zhigang

Chen, Zhixi

Chen, Zhongbing

Cheng, Chin Chi

Cheng, Ching-Hwa

Cheng, Chin-hsing

Cheng, Eric Ka-Wai

Cheng, Huanyu

Cheng, Jie

Cheng, Lin

Cheng, Pei

Cheng, Shouyun

Cheng, Tao

Cheng, Yu-Huei

Cherepovitsyn, Alexey

Chernyshov, Mikhail

Chesneau, Xavier

Cheung, Howard

Chi, Kai-Hsien

Chiacchio, Ferdinando

Chianese, Simeone

Chiang, Chen-Yu

Chiang, Yu-Chun

Chiappini, Daniele

Chiaraviglio, Luca

Chiba, Kazuhisa

Chien, Yu-Chien

Chih, Mingchang

Chikumbo, Oliver

Chin, Cheng Siong

Ching, T. W.

Chinnici, Alfonso

Chiodo, Elio

Chirathadam, Thomas Abraham

Chistoserdova, Ludmila

Chitale, Kedar
Chivaee, Hamid Sarlak

Cho, Dae Won

Cho, Younghoon

Cho, Youngsang

Choens, Robert Charles

Choi, Dae-Hyun

Choi, Deokjai

Choi, Dong Gu

Choi, Gyewoon

Choi, Jaeho

Choi, Jungwook

Choi, Minsuk

Choi, Nag Jung

Choi, Sangyup

Choi, Won-Chang

Choi, Wonjun

Choi, Woojin

Choi, Yosoon

Chong, Alvin

Choo, Kyosung

Choon-Man, Jang

Chou, Jung-Chuan

Chowdhury, Jahedul

Chrenko, Daniela

Christian, Eitzinger

Christodoulides, Paul

Christodoulou, Christos A.

Christoforidis, Georgios

Chrysikopoulos, Constantinos V.

Chrysoulas, Christos

Chu, Ka Kee Alfred

$\mathrm{Chu}, \mathrm{Yi}$

Chu, Yongbin

Chuah, Yew Khoy

Chuang, Abel

Chuang, Hui-Jen

Chubb, Lauren G.

Chui, Kwok Tai

Chun, Youngpil

Chung, Kung-Ming

Chung, Sheng-Heng

Chung, Y.N.

Chung, Ying-Chien

Ciancio, Virgilio

Ciani, Adriano

Cicala, Gianluca

Cicconi, Paolo

Cintuglu, Mehmet Hazar

Ciobanu, Radu Ioan

Cionca, Victor

Ciornei, Irina

Cipollone, Roberto

Cirimele, Vincenzo

CIRSTEA, Stefan

Ciuonzo, Domenico

Ciupitu, Liviu

Civiero, Paolo

Claridge, David 
Clarkson, Peter

Clausen, Philip

Coates, Colin

Cobben, J.F.G.

Coccia, Gianluca

Codina, Esteve

Coe, Ryan

Coiro, Domenico P.

Colangelo, Gianpiero

Colantoni, Andrea

Colla, Valentina

Collado, José Villar

Collins, Keri

Colombo, Emanuela

Colomer-Llinàs, Joan

Comes, Calin

Conceição, Eusébio Z.E.

Concli, Franco

Cong, Rong-Gang

Congedo, Paolo Maria

Congestri, Roberta

Connelly, Elizabeth

Constantinou, Michael

Conte, Francesco

Contestabile, Pasquale

Conti, Paolo

Corbí, Hugo

Corchia, Laura

Corcione, Massimo

Cordiner, Stefano

Cormos, Calin-Christian

Cornaro, Cristina

Cornea, Octavian

Cornélusse, Bertrand

Corral Bobadilla, Marina

Corsini, Alessandro

Corti, Enrico

Corvacho, Helena

Cosentino, Valentina

Costa Freitas, Maria De Belém

Costa, Carlos M.

Costa, Dulce

Costa, Mário

Costa, Paulo Moisés

Costa-Castelló, Ramon

Costanzo, Alessandra

Costanzo, Vincenzo

Cotfas, Liviu-Adrian

Cotfas, Petru

Cottafava, Dario

Coulter, John

Coxon, Paul

Cozzolino, Raffaello

Croonenbroeck, Carsten

Cruz-Romero, Pedro

Cuadras, Angel

Cubas, Javier

Cucchiella, Federica
Cucciniello, Raffaele

Cui, Borui

Cui, Fangda

Cui, Hantao

Cui, Longji

CUI, Miao

Cui, Mingjian

Cunha, Jorge

Curiac, Daniel

Curtis, John

Curtis, Robin

Cyganowski, Piotr

Cyklis, Piotr

Cyran, Katarzyna

Czech, Piotr

Czujko, Tomasz

Czumbil, Levente

D'Auria, Francesco

Dąbrowski, Wojciech

D'Agostino, Delia

Dagoumas, Athanasios

Dahle, Sebastian

Dai, Baiqian

Daidzic, Nihad

Daigle, Hugh

Dakka, Sam M.

Dalla Vedova, Matteo D. L.

Damartzis, Theodoros

Dambone Sessa, Sebastian

Damiano, Alfonso

Damma, Devaiah

Dan, Floricău

D'Andrea, Antonio

D'Andreagiovanni, Fabio

Danehkar, Ashkbiz

Dang, Minh Trung

Daniele, Elia

Dannier, Adolfo

Dao, Phong B.

Daraban, Stefan

Da-Ronch, Andrea

D'Arpino, Matilde

Darwish, Muge Mukaddes

Das, Manohar

Das, Narottam

Das, Oindrila

Datcheva, Maria

Datta, Rupsa

Davari, Masoud

Davide, Astolfi

Davidson, Josh

De Belie, Frederik

De Castro, Alexandre

De Cauwer, Cedric

De Falco, Pasquale

De Iuliis, Silvana

de Jong, Martin

De La Morena, Joaquin 
De La Torre Larrañaga, Unai

de Lacalle, Luis Norberto López

De Luca, Luigi

De Menna, Fabio

De Oliveira Santos, Lino

De Paepe, Ward

De Palma, Pietro

De Paola, Pierfrancesco

De Paoli, Marco

De Pertis, Marco

De Rosa, Mattia

De Santis, Michele

De Schepper, Guillaume

De Simone, Marco Claudio

De Simón-Martín, Miguel

De Tommasi, Luciano

De Tuglie, Enrico

Deangeli, Chiara

Deb, Chirag

Deb, Proloy

Degiannakis, Stavros

Dehghani Tafti, Hossein

Dehghani-Sanij, Alireza

Dehghanpour, Hassan

Deilami, Sara

Dejam, Morteza

Del Olmo Larrañaga, Jon

Del Pero, Francesco

Del Pizzo, Andrea

Delcea, Camelia

Delfs, Jens-Olaf

Delgado Prieto, Miguel

Delgoshaei, Parhum

Deligios, Paola A.

Delimar, Marko

Della Valle, Nives

Delle Rose, Marco

Dellnitz, Andreas

Demirocak, Dervis Emre

Demoulias, Charis

Denai, Mouloud

Denis, Proshlyakov

Denkenberger, David

Deppman, Airton

Depuru-Mohan, Karthik

Derrick, Mott

Deshmukh, Suhrid

Desmet, Bernard

Dettori, Marco

Dev, Soumyabrata

Devia, Francesco

Dewallef, Pierre

DeWilde, Pieter

Dey, Satadru

Dhakal, Dinesh

Dhaou, Riadh

Dharavath, Srinivas

Di Bartolomeo, Antonio
Di Battista, Davide

Di Cara, Dario

Di Franco, Francesco

Di Martino, Ferdinando

Di Nardo, Armando

Di Noia, Luigi Pio

Di Sarli, Valeria

Di Silvestre, Maria Luisa

Di Somma, Marialaura

Di Tommaso, Antonino Oscar

Diaconu, Daniel Constantin

Diallo, Demba

Diallo, Thierno M. O.

Dias, Frederic

Dias, Morgado

Diaz Gonzalez, Juan

Díaz Meneses, Gonzalo

Díaz-Hernández, Gabriel

Dickey, Bret

Dicorato, Maria

Didier, Gaetan

Dien, Bruce

Diez, David Borge

Diez, José M.

Digiesi, Salvatore

Dimakis, Nicholas

Dimitrakopoulos, Georgios

Dimitriou, Vasileios

Dinh, Nguyen

Dinis, Maria Alzira Pimenta

Dipasquale, Chiara

Dixit, Mudit

Dixon, David A.

Dizqah, Arash M.

Dodson, Elizabeth

Dolara, Alberto

Doleček, Petr

Dolega, Boguslaw

Dolinska, Silvia

Dombek, Grzegorz

Domenech, Bruno

Domine, Marcelo

Domingo Irigoyen, Silvia

Domingues, Nuno

Dominguez, Ruth

Dominguez, Sergio

Domínguez-García, José Luis

Domínguez-Navarro, José Antonio

Dominković, Dominik Franjo

Donaldson, A. Burl

Donateo, Teresa

Donchev, Alexander

Dong, Jianning

Dong, Zhizhong

Dopazo, Gonzalo Astray

Dormido, Raquel

Doroudiani, Saeed

Dos Santos, Rui Galhano 
Dósa, György

Dotzauer, Erik

Doudin, Khalid

Doujak, Eduard

Doulos, Lambros $\mathrm{T}$.

Doval-Gandoy, Jesus

Dovì, Vincenzo

Doytch, Nadia

Drabczyk, Kazimierz

Dreżewski, Rafał

Drillet, Jean-François

Druica, Elena

Du, Jiangtao

Du, Peng

$\mathrm{Du}$, Ya-Ping

$\mathrm{Du}$, Yuncheng

Duan, Dongliang

Duan, Nan

Duarte, Amílcar

Dubey, Ashish

Dubey, Rameshwar

Duchesne, Marc

Ducoing Ruiz, Cristián A.

Duda, Mandy

Duda, Piotr

Dudyński, Marek

Dudziak, Tomasz

Duffy, Alistair

Dulebenets, Maxim A.

Dumas, Jean-Pierre

Dumbrava, Stefan

Dumbrava, Virgil

Dumitran, Laurentiu

Dumitran, Laurențiu Marius

Dumitras, Diana

Dumitrescu, Ana-Maria

Dumitrescu, Mariana

Dunai, Larisa

Dunea, Daniel

Duong, Hung Cong

Duong, Minh Quan

Duong, Trung

Dutta, Rukmi

Dyatkin, Boris

Dyjakon, Arkadiusz

Dykas, Sławomir

Dylewski, Robert

Dziurdzia, Piotr

Dziurzński, Wacław

Eberle, Ulrich

Eble, Cortland

Ebrahimi, Amin

Effiom, Samuel Oliver

Efimova, Elena

Eftekhar Azam, Saeed

Eftekhari, Behzad

Eguchi, Kei

Eiksund, Gudmund Reidar
Eilts, Peter

Ejdys, Joanna

Ekonomou, Lambros

Ekpeni, Leonard

El Mejdoubi, Asmae

Eldeeb, Mazen

Elduque, Daniel

Eleni, Didaskalou

El-Halwagi, Mahmoud

Ellahi, R

Elmasidis, Konstantinos

Elorza, Iker

Elsaiah, Salem

El-Shahat, Adel

Elsner, Paul

Emad, Muhammad Zaka

Emberley, Richard

Emmanuel, Kakaras

Emodi, Nnaemeka

Endo, Patricia Takako

Enescu, Diana

Entezami, Mani

Epelde, Eva

Erdem, Emre

Erdem, Ergin

Erdogan, Nuh

Erhart, Tobias

Erickson, Larry

Ericson, Liselott

Ericsson, Karin

Escolano, Sergio Orts

Escudero Gómez, Luis Alfonso

Eslami, Hossein

Esteban, M. Dolores

Estelle, Patrice

Estêvão, João M.C.

Esteves, Eduardo

Estévez, Francisco José

Etacheri, Vinodkumar

Etemaddar, Mahmoud

Etienne, Xiaoli

Euiseok, Hwang

Eustance, Everett

Everett, Vernie

Eydani Asl, Mohamad

Fabbri, Kristian

Facão, Jorge

Facci, Andrea

Fahes, Mashhad

Fai A. Lo, Kwong

Failla, Giuseppe

Fajri, Poria

Falcone, Pasquale Marcello

Faleiro, Eduardo

Falenty, Andrzej

Faleschini, Flora

Falkowski, Piotr

Fan, Guodong 
Fan, Rui

Fan, Wenke

Fan, Xiaoyuan

Fandi, Ghaeth

Fang, Shuyu

Fang, Wei-Ta

Fang, Yi

Fara, Laurentiu

Farahmand, Kambiz

Fargnoli, Mario

Farhadi, Akram

Faria Albanese, Jimmy Alexander

Faria, Pedro

Farquharson, Jamie

Farrell, Michael

Farrokhabadi, Mostafa

Farrokhpanah, Amirsaman

Farroni, Flavio

Favuzza, Salvatore

Fayaz, Mohammadreza

Fayazbakhsh, Mohammad Ali

Fegade, Swapnil

Feijóo, Andrés

Feito, Norberto

Fekete, Krešimir

Fekete, Mária Magdolna

Felgueiras, Carlos

Feliziani, Mauro

Felsmann, Clemens

Feng, Cai

Feng, Huicheng

Feng, Ruimin

Feng, Wei

Feng, Xianyong

Feng, Yin

Feng, Yongcun

Ferdyn-Grygierek, Joanna

Fernandes, Fábio

Fernández Bandera, Carlos

Fernández, Julio Blanco

Fernández-Agüera, Jessica

Fernández-Cardador, Antonio

Fernandez-Gamiz, Unai

Fernandez-Garcia, Javier

Fernández-Guillamón, Ana

Fernandez-Morales, Francisco Jesus

Fernández-Oro, Jesús Manuel

Fernando, Ana Luisa

Fernando, Nuwantha

Ferrari, Michele

Ferrarini, Giovanni

Ferreira, Joao

Ferreira, Joao C.

Ferreira, Joao Carlos Amaro

Ferreira, Jorge

Ferreira, Paulo

Ferrer, Esteban

Festa, Demetrio Carmine
Ficarella, Antonio

Ficco, Giorgio

Fidelibus, Corrado

Fiebig, Wiesław

Fiedler, Brian $\mathrm{H}$.

Figari, Massimo

Figlus, Tomasz

Figueroa Meza, Maria Josefina

Filanovsky, Igor

Filipowicz, Mariusz

Filizadeh, Shaahin

Finck, Steffen

Finkelman, Robert

Finšgar, Matjaž

Fioreze, Tiago

Fiori, Anna Maria

Fiori, Luca

Fiorito, Francesco

Fisch, Michael

Fister, Iztok

Floors, Rogier

Flores-Arias, Jose-Maria

Florez, Horacio

Florian Baron, Camilo

Florinda, Figueiredo Martins

Flourentzou, Nikolas

Flox, Cristina

Fly, Ashley

Fogarassy, Csaba

Foglia, Giovanni Maria

Fokaides, Paris

Folea, Silviu

Folley, Matt

Fonseca I Casas, Pau

Fontani, Daniela

Fopah-Lele, Armand

Forooghi, Pourya

Förster, Anna

Forte, Giovanni

Fortuna, Luigi

Fosso, Olav

Fouladirad, Mitra

Fowler, Michael

Fraga-Lamas, Paula

Fragos, Vassilios

Fraile, José M.

Francavilla, Matteo

Francik, Sławomir

Francisco José, Sánchez Sutil

Franke, Jörg

Franzitta, Vincenzo

Frauke, Urban

Frazzica, Andrea

Freddi, Alessandro

Fredianelli, Luca

Freire, Fausto

Frese, Christian

Freund, Jonathan B. 
Frey, Georg

Friedrich, Christoph M.

Friesen, Alex

Fritsche, Uwe

Frivaldsky, Michal

Frontera, Patrizia

Frontistis, Zacharias

Frosina, Emma

$\mathrm{Fu}$, Weinong

$\mathrm{Fu}$, Xingang

$\mathrm{Fu}$, Xuebing

$\mathrm{Fu}$, Yangya

$\mathrm{Fu}$, Zhezhen

Fuentes-Bargues, José Luis

Fuertes-Miquel, Vicente S.

Fujii, Yoshiaki

Fujimoto, Yu

Fujisawa, Tomotsumi

Fulginei, Francesco Riganti

Funck, Torsten

Fuoco, Alessio

Fura, Barbara

Futcher, Julie

G. Speight, James

Gabaldón, Antonio

Gabaldon-Estevan, Daniel

Gabash, Aouss

Gabbar, Hossam A.

Gabbrielli, Roberto

Gabl, Roman

Gabriela, Dobrotă

Gabriele, Grandi

Gadaguntla Radhakrishnan, Balachandran

Gagliano, Antonio

Gagne II, David John

Gago-Calderón, Alfonso

Gaiceanu, Marian

Gajowniczek, Krzysztof

Galatioto, Alessandra

Galdeano-Gómez, Emilio

Galeani, Sergio

Galić, Mario

Galipeau, David W

Galla, Stanislaw

Gallego, Jesús Ángel Román

Galletti, Anna Maria Raspolli

Gallo, Carmela

Galvão, João

Galvão, João Rafael

Gamboa, Paulo José Duarte Landeiro

Gamez-Montero, P.J.

Gamez-Montero, Pedro Javier

Gammon, Tammy

Gamo, Javier

Gamwo, Isaac K.

Gan, Quan

Gan, Yong X.

Ganea, Daniel
Ganguly, Sayantan

Gao, David

Gao, Fei

Gao, Leiming

Gao, Shangce

Gao, Tongchuan

Gao, Wenpei

Gao, Yabiao

Gao, Yuan

Garapati, Nagasree

Garbaras, Andrius

Garbin, Enrico

Garcerá, Gabriel

Garcia Bermejo, Juan T.

García Gil, Rafael

Garcia, Belen

García, Carmelo

García, Carmelo R.

Garcia, Jordi

Garcia, José M.

García, Manuel Pérez

García, Mario Ortiz

García, Miguel Torres

Garcia, Rosario

Garcia-Cerrada, Aurelio

García-Contreras, María Reyes

García-González, Javier

García-Gusano, Diego

Garcia-Saavedra, Andres

García-Triviño, Pablo

García-Vázquez, Carlos Andrés

Gardy, Jabbar

Garg, Akhil

Gargano, Francesco

Garicano-Mena, Jesús

Garrido-Jurado, Juan

Garrigós, Ausias

Garrote, Gil

Garvey, Seamus D.

Gatteschi, Valentina

Gatto, Gianluca

Gavouneli, Maria

Gavrilas, Mihai

Gawin, Dariusz

Ge, Baoming

$\mathrm{Ge}$, Cui

Ge, Haiwen

Ge, Xumeng

Gębicki, Jacek

Gecevičius, Giedrius

Gejji, Rohan

Gelbstein, Yaniv

Gelegenis, John

Gels, Stefan

Geng, Bo

Geng, Linxiao

Genty, Eric

Georgakellos, Dimitrios A. 
George, Thomas F.

Georgescu, Sanda-Carmen

Gerard, Mathias

Gercek, Cihan

German, Konstantin E.

Ghamari, Mohsen

Ghanbari, Niloofar

Ghanbarian, Behzad

Ghasemi-Fare, Omid

Ghassemi, Mona

Ghatak, Kamalika

Ghazi Wakili, Karim

Gheorghe, Grigoras

Gheorghiu, Razvan Andrei

Gherghina, Ştefan Cristian

Ghezehei, Solomon

Ghiani, Emilio

Ghiaus, Christian

Ghidelli, Matteo

Ghodke, Chaitanya

Ghofrani, Mahmoud

Gholamalizadeh, Ehsan

Ghosh, Aritra

Ghosh, Prasanta

Ghosh, Tathagata

Ghoshal, Shraboni

Giani, Annarita

Giannakopoulos, Dionysios

Giasin, Khaled

Gibson, Richard

Gidaspow, Dimitri

Giernacki, Wojciech

Giglio, Emanuele

Gil, Antoni

GIl, Bartosz

Gil, Ignacio Hernando

Gil, M. Victoria

Gil, Nuno J.

Gilardi, Elisa

Gilart-Iglesias, Virgilio

Gil-de-Castro, Aurora

Gilroyed, Brandon

Gimelli, Alfredo

Gingerich, Daniel B.

Giorgio, Guariso

Giral, Roberto

Giudici, Mauro

Giungato, Pasquale

Giurca, Ioan

Gładyszewska-Fiedoruk, Katarzyna

Glassley, William E.

Glassley, William Edward

Glowacz, Adam

Glüsen, Andreas

Glushkov, Dmitrii

Glynn, Peter J.

Gockenbach, Ernst

Godina, Radu
Göksu, Ömer

Golasi, Iacopo

Goldfarb, Jillian L.

Goldoni, Emanuele

Goldstein, Rachel Rosenberg

Goli, Preetham

Golob, Marjan

Golter, Paul

Gomes, João Fernando Pereira

Gómez Barrios, Xiomar

Gómez-Calvet, Roberto

Gómez-Hernández, Jesús

Gomez-Lazaro, Emilio

Gomez-Romero, Juan

Gómez-Soberón, José Manuel

Gonçalves, David E. P.

Goncalves, Olivier

Gontean, Aurel Stefan

Gonzalez Crespo, Ruben

Gonzalez De Durana, José María

González De La Rosa, Juan José

González Pérez, Isaías

González, Alejandro

Gonzalez, Camino

Gonzalez, Luis Sanchez

Gonzalez-Longatt, Francisco

Gonzalez-Medina, Raúl

González-Romera, Eva

Goodarzi, Marjan

Goodyer, Eric

Goosey, Emma

Gopalakrishnan, Bhaskaran

Gopalan, Sai Anand

Gopisetty, Satya

Goranovic, Goran

Gordon, Robert

Gorji, Saman

Gormally, Alexandra

Górnicki, Krzysztof

Górski, Marcin

Gosiewski, Krzysztof

Goto, Kazuya

Götz, Annette E.

Gou, Zhonghua

Goude, Anders

Goupee, Andrew

Grabowski, Marcin

Grachev, Andrey

Graditi, Giorgio

Grande, Carlos

Grasso, Francesco

Grasso, Marzio

Gray, Murray

Green, Robert C.

Greene, Scott

Greenman, John

Greenough, Rick

Greenwood, David 
Gregov, Goran

Greiner, Martin

Grela, Jakub

Grimm, Alejandro

Gritli, Yasser

Griva, Giovanni

Grondzik, Walter

Groot, Jens

Grossi, Marco

Grosu, Yaroslav

Grothues, Thomas

Gryning, Mikkel Peter Sidoroff

Gryning, Sven-Erik

Grzejszczak, Piotr

$\mathrm{Gu}$, Wenting

Guan, Wenjian

Guariglia, Emanuel

Guarini, Maria Rosaria

Guariso, Giorgio

Gudmundsson, Snorri

Gueorguiev, G.K.

Guerra, Omar

Guerrero, Juan I.

Gui, Yonghao

Guilbert, Damien

Gulagi, Ashish

Gulgunje, Prabhakar

Gulkowski, Slawomir

Gullo, Paride

Guntur, Srinivas

Guo, Liejin

Guo, Qingbo

Guo, Xiaofeng

Guo, Xinfei

Guo, Youguang

Guran, Serpil

Gutiérrez, Aaron

Guzman, Marcelo

Guzman, Ramon

Gwozdziewicz, Maciej

Gyore, Attila

Gyselinck, Johan

Ha, Sung Kyu

Haas, Francis

Haddad, Brent

Haddad, Sofiane

Haes Alhelou, Hassan

Hafezi, Hossein

Hagnestål, Anders

Hahn, Konstanze R.

Hailu, Getu

Hajizadeh, Amin

Halawa, Edward

Halios, Christos

Halisch, Matthias

Hall, Matthew

Hall, Monika

Hallil, Hamida
Hamawand, Ihsan

Hamed, Ali

Hamed, Ali M.

Hamidi, Reza

Hamidi, Seyed Ahmad

Hamiduddin, Iqbal

Hamilton, Nicholas

Hammami, Manel

Hammerstrom, Donald J.

Hamori, Shigeyuki

Hampton, Jennifer

Hamza, Neveen

Han, Se-Kyung

Han, Wenlin

Han, Won-Sik

Haneklaus, Nils

Hanly, Jim

Hann, Christopher

Hannouf, Marwa

Hanquist, Kyle

Hansen, Alex

Hansen, Jason

Hansen, Jeffrey

Hansen, Niels

Hansen, Nils

Hansen, Tim

Hao, Guangbo

Hao, Yong

Haq, Rizwanul

Hara, Yutaka

Hariri-Ardebili, Mohammad Amin

Harnefors, Lennart

Harrington, Julie

Harris, Stephen J.

Hart, Abarasi

Hartmann, Ingo

Hartvigsson, Elias

Hasager, Charlotte

Hasan, Md Mubashwar

Haseli, Yousef

Hasiuk, Franciszek

Hasmatuchi, Vlad

Hassan Khattak, Sanober

Hassan, Firas

Hassanin, Hany

Hatziadoniu, Constantine

Hatziargyriou, Nikolaos

Hatzikraniotis, E.

Hausberger, Stefan

Hauser, Michael

Hazari, Md. Rifat

He, Miao

He, Peiwen

Healy, William M.

Hebner, Robert

Hedayati, Ali

Hegedűs, László

Heidarinejad, Mohammad 
Heidler, Fridolin

Heinemann, Niklas

Heinonen-Tanski, Helvi

Helena, Navarro

Helmut, Yabar

Helseth, Lars

Heltzel, Jacob

Hemanathan, Kumar

Henao, Nilson

Hendrix, Marcel

Henke, Markus

Henrie, Morgan

Henriques, C. Oliveira

Hensgen, Frank

Herbei, Mihai Valentin

Herbes, Carsten

Hermans, Thomas

Hermoso-Orzáez, Manuel Jesús

Hernandez, Jesus C.

Hernández-Callejo, Luis

Hernik, Bartłomiej

Heron, Graeme

Herrando, María

Herranz, Raul Cano

Herrera, Luis

Herrero, L. Carlos

Herrmann-Geppert, Iris

Hesaraki, Arefeh

Hester, Keith

Hidalgo, Arturo

Hiernaux, Stéphane

Higinio, Sánchez Sainz

Hihara, Eiji

Hilber, Patrik

Hill, Graeme

Hill, Josephine Mary

Hiraishi, Tetsuya

Hirano, Y.

Hittinger, Eric

Hiziroglu, Huseyin

Hjertager, Bjørn

Hnydiuk-Stefan, Anna

Ho, Tan-Jan

Ho, Wen-Jeng

Hoblos, Ghaleb

Hobosyan, Mkhitar

Hoecker-Martinez, Martin

Hoekman, S. Kent

Hoelling, Michael

Hofer, Matthias

Hoff, Craig J.

Hofmann, Mathias

Höger, Marek

Hojat Jalali, Himan

Hokamoto, Kazuyuki

Holderbaum, William

Holopainen, Riikka

Hołub, Marcin
Holvad, Torben

Homola, Tomáš

Hong, Liang

Hong, Min

Hong, Seung Ho

Hong, Wei-Chiang

Hönig, Vladimír

Honkonen, Tuula

Honrubia-Escribano, Andres

Honus, Stanislav

Hoque, Enamul

Horn, Susanna

Horng, Shih-cheng

Hős, Csaba

Hossain, Eklas

Hossain, Eklass

Hossain, Jahangir

Hosseininoosheri, Pooneh

Hosseinzadeh, Mehdi

Hou, Shuhn-Shyurng

Housiadas, Kostas

Howieson, Stirling G.

Howlader, Abdul Motin

Hoznedl, Michal

Hrad, Jaromir

Hren, Alenka

Hsiao, Chun-Ching

Hsiao, H.L.

Hsieh, Bieng-Zih

Hsieh, Hsin-Hsin

Hsieh, Min-Fu

Hsu, Ming-Wei

$\mathrm{Hu}$, Aiguo Patrick

$\mathrm{Hu}$, Bo

$\mathrm{Hu}$, Fengkai

$\mathrm{Hu}$, Jia-Sheng

$\mathrm{Hu}$, Jin-Li

$\mathrm{Hu}$, Maocong

$\mathrm{Hu}$, Ming-Che

$\mathrm{Hu}$, Weifei

$\mathrm{Hu}$, Xiaosong

$\mathrm{Hu}$, Yihua

$\mathrm{Hu}$, Yong

$\mathrm{Hu}$, Yukun

$\mathrm{Hu}$, Zhiwei

Huang, Cheng-Tsu

Huang, Chung-Neng

Huang, Gordon

Huang, Hsin-Haou

Huang, Jenny

Huang, Jingfeng

Huang, Nantian

Huang, Pei-Hwa

Huang, Sunan

Huang, Tao

Huang, Wei-Tzer

Huang, Wu-Jang

Huang, Xingfeng 
Huang, Yanjun

Huang, Yu-Hsi

Hugi, Christoph

Huh, Jung-Ho

Huh, Jun-Ho

Huh, Jun-Ho Huh

Hui, Ron S.Y.

Huminic, Gabriela

Hung, Tai-Feng

Hur, Deog Jae

Hur, Jin

Huria, Tarun

Hurink, Johann

Hussain, Amjad

Hussain, Babar

Hussain, Ghulam Amjad

Hussain, Iftikhar

Hussain, Muhammad

Hussain, S.M. Suhail

Hwang, Bon-Gang

Hynes, Mike

Iannuzzo, Francesco

Ibanez, Federico Martin

Ibarra-Berastegi, Gabriel

Ibrahim, Hussameldin

Ichinose, Toshiaki

Idiano, D'Adamo

Idsø, Johannes

Ifelebuegu, Augustine Osamor

Ihara, Takeshi

Ikegami, Masashi

Ikegaya, Naoki

Ikeo, Shigeru

Ilves, Risto

Im, Jeongdae

Im, Seong-Kyun

Inagaki, Atsushi

Inard, Christian

Incremona, Gian Paolo

Infield, David

Insausti, Xabier

Invernizzi, Costante Mario

Ioakimidis, Christos

Ioannidis, Dimosthenis

Ioannidis, Georgios

Iodice, Paolo

Ioelovich, Michael Ya

Ion, Catalin Petrea

Iorgulescu, Mariana

Ipsakis, Dimitris

Irace, Andrea

Irannejad, Abolfazl Mani

Iribarren, Diego

Ishihara, Keiichi N.

Islam, Mohammad

Islam, Shama Naz

Ismail, Tareq

Ismail, Umair
Iuppa, Claudio

Ivancic Santek, Mirela

Ivanov, Valentin

Iyer-Raniga, Usha

Iyota, Taketoshi

Izacard, Olivier

Jabłoński, Marek

Jacopo, Bacenetti

Jacques, Sebastien

Jacyna, Marianna

Jafari, Raheleh

Jafari, Soheil

Jafarinejad, Shahryar

Jaganathan, Arun

Jäger, Alexander

Jagiela, Mariusz

Jahromi, Hossein

Jain, Ankur

Jaiswal, Amit K.

Jakóbik-Kolon, Agata

Jakus, Damir

Jampaiah, Deshetti

Jamrozik, Arkadiusz

Jang, Jerhuan

Jankovic, Ljubomir

Janusas, Giedrius

Jaramillo-Morán, Miguel A.

Jason, Ede

Jauch, Clemens

Javaherchi, Teymour

Javanbakht, Gina

Jayakumar, Arunkumar

Jayamohan, Harikrishnan

Jayaraman, Ambal

Jayasinghe, Shantha

Jean, Fu-Rong

Jelley, Nick

Jen, Shien Uang

Jeng, Tzer-Ming

Jeon, Eui-Chan

Jeong, Jae-Weon

Jeong, Jin Su

Jeong, Soo Kim

Jeong, Yeonho

Jesenik, Marko

Jesus, Isabel

Jeung, Sung-Hwa

Jeyasurya, Benjamin

Jezierski, Jan

Jha, Pankaj

Ji, Bing

Ji, Jinchen

Ji, Xiaobo

Ji, Yingchun

Jia, Bao

Jia, Boru

Jia, Charles

Jia, Fanli 
Jia, Junbo

Jia, Yu

Jiang, Huaiguang

Jiang, Liben

Jiang, Ruinian

Jiang, Wenbin

Jiang, Zhiyu

Jianu, Ionel

Jianu, Iulia

Jimenez, Emilio

Jimenez, Felipe

Jiménez, José A.

Jimenez-Bescos, Carlos

Jin, Xinfang

Jitea, Ionel Mugurel

Jo, Darkwa

Jobbágy, Ján

Jocher, Agnes

Johansson, Maria T.

Johnson, Brian

Johnson, David

Johnson, Erick

Johnson, Thomas

Johnston, Steven

Jonek-Kowalska, Izabela

Jongerden, Marijn R.

Jonsson-Niedziolka, Martin

Jorge, Celeste

Joshi, Anshul

Joshi, Niravkumar

Joshi, Sanket

Jotshi, Chand

Jovanović, Slaviša

Józsa, Viktor

Juaidi, Adel

Julien, Christian M.

Julrat, Sakol

Jun, Sunghae

Jung, Minchae

Jung, Seungmin

Jung, Sung Kwon

Jung, Sunghun

Junghans, Lars

Junyent-Ferré, Adrià

Jurado, Francisco

Jurasz, Jakub

Jurca, Florin

Jureczko, Mariola

Jurikova, Tunde

Justino, Paulo

Justo, Jackson John

Kabanos, Themistoklis

Kåberger, Tomas

Kabiersch, Grit

Kabitzsch, Klaus

Kacem, Najib

Kaczmarek, Michał

Kahrobaee, Salman
Kaiwartya, Omprakash

Kajtaz, Mladenko

Kalambura, Sanja

Kalantari, Amirmasoud (Masoud)

Kalla, Smail

Kalligeros, Stamatis Spyridon

Kalogiannis, Konstantinos G.

Kalsi, Swarn

Kalt, Gerald

Kamali, Fatemeh

Kambezidis, Harry D.

Kaminski, Marcin

Kamiński, Marcin

Kamsu-Foguem, Bernard

Kan, Eunsung

Kanarachos, Stratis

Kandasamy, Nandha Kumar

Kanellopoulos, Christos

Kanetis, Loukas

Kang, He-Yau

Kang, Jimyung

Kang, Min Gyu

Kang, Ning

Kang, Sanggyu

Kani, Ali Pourmousavi

Kania, Dariusz

Kanter, Theo G.

Kantzas, Apostolos

Kapadia, Rehan

Kapcia, Konrad

Kaplanis, Socrates

Kappatou, Joya C.

Kappera, Rajesh

Kapsalis, Vasilios C.

Kapsalis, Vassilis

Karagiannakis, George

Karakatič, Sašo

Karazhanov, Smagul

Karbaschian, Mohammad Ali

Karimi, Mazaher

Karimi-Ghartemani, Masoud

Karipidis, Filippos

Karlin, Ilya

Karoń, Grzegorz

Karra, Satish

Kartopu, Giray

Karuppasamy, Kannan

Kashimura, Keiichiro

Kasperski, Jacek

Kasprzycka, Agnieszka

Kassanos, Panagiotis

Kataoka, Yasuhiro

Kateris, Dimitrios

Katsigiannis, Yiannis

Katunsky, Dusan

Katz, Jonathan I.

Kaur, Aman Preet

Kawalec, Adam 
Kayukova, G. P.

Kazak, Andrey

Kazakbaev, Vadim

Kazama, Toshiharu

Kazda, Tomáš

Kazemi, Mohammad

Keilegavlen, Eirik

Kelly, Geoff

Keogh, Eamonn

Kerdphol, Thongchart

Keshavarzian, Sajjad

Kew, Peter Arthur

Khachatryan, Lavrent

Khadilkar, Aditi

Khadka, Dhruba B.

Khalid, Farrukh

Khaliq, Jibran

Khambadkone, Ashwin M.

Khan, Md Tanvir Arafat

Khan, Muhammad

Khan, Sohail

Kharrazi, Ali

Khataniar, Santanu

Khatib, Tamer

Khazaei, Javad

Khodaparast, Jalal

Khojasteh Salkuyeh, Yaser

Khormali, Shahab

Khoshmanesh, Khashayar

Khushalani Solanki, Sarika

Kiani, Mehdi

Kiefer, Johannes

Kiefer, Rudolf

Kies, Alexander

Kiesewetter, Dmitry

Kikas, Timo

Kil, Gyung-Suk

Kim, Albert

Kim, Byungtaek

Kim, Changhee

Kim, Cheorl-Ho

Kim, Chunghun

Kim, Daehwan

Kim, Eui Jong

Kim, Hanki

Kim, Hee-Je

Kim, Heejin

Kim, Hee-Seok

Kim, Heuy Dong

Kim, Hongsuk

Kim, Ho-Yeon

Kim, Hye-Mi

Kim, Hyunsoo

Kim, Jaehong

Kim, Jae-Kon

Kim, Jang Mok

Kim, Jinsoo

Kim, Jung Kyu
Kim, Jung Rae

Kim, Jungsu

Kim, Katherine

Kim, Kibum

Kim, Kwang Sik

Kim, Kyeong-Hwa

Kim, Kyeongseok

Kim, Kyu-Hong

Kim, Kyung Chun

Kim, Min Soo

Kim, Min-Hwi

Kim, Mun-Kyeom

Kim, Sanghoek

Kim, Seok-Kyoon

Kim, Seoyong

Kim, Seung Wan

Kim, Soo Young

Kim, Sunghyun

Kim, Sung-Koo

Kim, Sun-Sook

Kim, Taehoon

Kim, Taesic

Kim, Wonhee

Kim, Youngbok

Kimoto, Sayuri

Kimura, Osamu

Kindl, Vladimir

King, Laurie

King, Peter

Kircher, Manfred

Kirchner, Mathias

Kireev, Dmitry

Kirke, Brian

Kisielewicz, Tomasz

Kisilewicz, Tomasz

Kitagawa, Toshiaki

Kitahara, Tatsumi

Kitajou, Ayuko

Kivalov, Sergey N

Klaić, Zvonimir

Klassen, Viktor

Kleimaier, Alexander

Klein, Tony

Klemeš, Jiří Jaromír

Klimach, Harald

Klimeš, Lubomír

Kloker, Markus J.

Klumpp, Matthias

Klvac, Radomir

Knap, Vaclav

Knápek, Alexandr

Knežević, Goran

Knittel, Dominique

Knoshaug, Eric

Knothe, Gerhard

Knowles, Mike

Ko, Byoung Chul

Ko, Chia-NaN 
Kobayashi, Makiko

Kobayashi, Noriyuki

Kofoworola, Oyeshola

Koirala, Binod

Koirala, Binod Prasad

Koji, Yamamoto

Kokkinos, Nikolaos

Kolaitis, Dionysios I.

Kolar, Vaclav

Kolasiński, Piotr

Kollár, László

Kolomenskiy, Dmitry

Koltsaklis, Nikolaos

Komori, Mochimitsu

Konar, Sumit

Kondo, Kayoko

Konist, Alar

Kononovicius, Aleksejus

Könözsy, László

Konstanciak, Anna

Konstantakopoulou, Foteini

Konstantakopoulou, Fotini

Konstantinou, Georgios

Konstantzos, Iason

Kontargyri, Vassiliki

Kontes, Georgios D.

Kontou, Eleftheria

Kopyscinski, Jan

Kor, Ah-Lian

Koraz, Yahya

Korbel, Jan

Korbel, Piotr

Koreanschi, Andreea

Korkali, Mert

Kornhuber, Stefan

Kortnik, Jože

Korzec, Marek

Korzeniewski, Marek

Koschorreck, Matthias

Kose, Utku

Kosmadakis, George

Kosnik, Lea-Rachel

Kosova, Klara

Kosovic, Branko

Koteich, Mohamad

Kotousov, Andrei

Kotsakis, Evangelos

Kotter, Richard

Kouchaki, Samaneh

Koukouzas, Nikolaos

Kováŕík, Ondřej

Kowalczyk, Sebastian

Kowalska, Ewa

Kowalski, Gregory

Koyama, Masato

Krah, Jens Onno

Kramer, Louisa

Krasuski, Adam
Kravari, Kalliopi

Krawczyk, Dorota Anna

Krejcar, Ondrej

Kricka, Tajana

Krim, Fateh

Krishnaswami, Hariharan

Kriston, Akos

Krisztin, Tamás

Križan, Peter

Krizek, Kevin

Krogerus, Tomi

Kroglund, Frode

Król, Małgorzata

Krolczyk, Grzegorz

Kronberg, Alexander

Krumdieck, Susan

Kruse, Andrea

Ktena, Aphrodite

$\mathrm{Ku}$, Cheng-Yu

Kuang, Shibo

Kubečková, Darja

Kubota, Keiichi

Kučera, Marián

Kucuksari, Sadik

Kudelcik, Jozef

Kuester, Matthew

Kufeoglu, Robin Sinan

Kujawski, Wojciech

Kukli, Kaupo

Kukulka, David J.

Kuleshov, Yuriy

Kumano, Teruhisa

Kumar, Asheesh

Kumar, Gautam

Kumar, Narendra

Kumar, Pramod

Kumar, Prasun

Kumar, Vivek

Kundu, Manab

Kuniewski, Maciej

Kuo, Cheng-Chien

Kuo, Jian-Long

Kuok, Sin-Chi

Kuperman, Alon

Kuravi, Sarada

Kurevija, Tomislav

Kuriqi, Alban

Kurnitski, Jarek

Kus, Vaclav

Kusch-Brandt, Sigrid

Kushkevych, Ivan

Kusiak, Dariusz

Kuti, Olawole

Kuyukina, Maria S.

Kuzle, Igor

Kvamme, Bjørn

Kvasov, Dmitri E.

Kwiecień, Iwona 
Kwon, Beomjin

Kwon, Joseph

Kwon, Minhae

Kyazze, Godfrey

Kyne, Dean

Kyratsis, Panagiotis

Kyriakarakos, George

Kyriakopoulos, Grigorios L.

La Rocca, Rosa Anna

Laaksonen, Hannu

Labidi, Jalel

Labovsky, Alexander E.

Lad, Jai

Ladero Galán, Miguel

Laes, Erik

Lafoz, Marcos

Łagód, Grzegorz

Lahaye, Domenico

Lai, Ching-Ming

Lai, Chiu-Keng

Lajos, Nagy

Lalizel, Gildas

Lam, John

Lam, K.H.

Lamarche, Louis

Lamberti, Alfredo

Lamedica, Regina

Landeka Dragičević, Tibela

Landi, Daniele

Landis, Florian

Langari, Reza

Lantz, Frédéric

Laopoulos, Theodore

Lappalainen, Kari

Lapthorn, Andrew Craig

Lara, David

Larosa, Claudio

Laskurain Iturbe, Iker

Latif, Eshrar

Latinopoulos, Charilaos

Latorrata, Saverio

Latosińska, Jolanta

Laudani, Antonino

Laudani, Antonio

Laukkanen, Timo

Laura, Moretti

Laureti, Tiziana

Laurini, Eleonora

Lautala, Pasi

Lavandera, Iván

Lawrence, Thomas M.

Lawrynczuk, Maciej

Lazari, Antonis

Lazaridis, Pavlos

Lazaroiu, George Cristian

Lazarou, Stavros

Le Besnerais, Jean

Le Clainche, Soledad
Le, Anh Dung Tran

Le, Tuan Anh

Le, Tuyen Quang

Leach, Felix

Leahy, Kevin

Leary, Peter

Łebkowski, Andrzej

Leblebici, Mumin Enis

Leccese, Fabio

Leccese, Francesco

Ledwon, Przemyslaw

Lee, Byongjun

Lee, ByoungHee

Lee, Changhee

Lee, Cheng-Ming

Lee, Chih-Hao

Lee, Christopher H. T.

Lee, Chun-Yao

Lee, Dong-Yeon

Lee, Duehee

Lee, Gee-Soo

Lee, Hyoungsoon

Lee, Hyunjin

Lee, Il-Oun

Lee, Jae Suk

Lee, Jaesuk

Lee, Jaewook

Lee, Jangmyung

Lee, Jeng-Dao

Lee, Jeong Ik

Lee, Johng-Ihl

Lee, Jong-Ha

Lee, Joon Kyu

Lee, Kui-Jun

Lee, Kyubock

Lee, Ming-Tsang

Lee, Moo-Yeon

Lee, Sang-Mae

Lee, Seok Woo

Lee, Su-Yol

Lee, Sze Sing

Lee, Taehwa

Lee, Woojoo

Lee, Yangsun

Lee, Yih-Der

Lee, Yong-Gil

Leger, Jean-Michel

Lei, Gang

Leibovici, Didier

Leijon, Jennifer

Leitão, João

Leiva, Fabricio

Lelea, Dorin

Lemu, Hirpa G.

Len, Christophe

Lennartsson, Patrik R.

Lenzo, Basilio

Leocadio, Hontoria García 
León, Gerardo A.

Leong, Jik-Chang

Leonowicz, Zbigniew

Leopardi, Angelo

Leporini, Mariella

Leu, Jun-Der

Lev, Dan

Leva, Sonia

Levihn, Fabian

Levron, Yoash

Lewandowska, Katarzyna

Lewandowski, Mirosław

Lewis, Randy S.

Lezama, Fernando

Li, Chong

Li, Dan

Li, Gen

Li, Guang

Li, Guang-Jin

Li, Guiqiang

Li, Hailin

Li, Hailong

Li, Hangyu

Li, Hao

Li, Hongkun

Li, Hua

Li, Jianwei

Li, Jie

Li, Jinchao

Li, Kai Yuan

Li, Liang

Li, Lijuan

Li, Meichun

Li, Mingxing

Li, Ning

Li, Peng

Li, Qifeng

Li, Qingan

Li, Rachel (Huijun)

Li, Rui

Li, Shuhui

Li, Silong

Li, Simon

Li, Steven

Li, Xianglin

Li, Xue

Li, Xuemin

Li, Xuesong

Li, Yangmin

Li, Yaping

Li, Yunchao

Li, Yuwei

Li, Zhi

Li, Zhiyi

Li, Zukui

Liang, An

Liang, Chao

Liang, Jun
Liang, Jun-Rui

Liang, Kun

Liang, Yi

Liang, Yucang

Liberati, Francesco

Liberato, Margarida

Libra, Martin

Lie, Tek-Tjing

Likozar, Blaž

Lillo-Bravo, Isidoro

Lim, Hyuk

Lim, Jae-Han

Limmer, Steffen

Lin, Bor-Ren

Lin, Chih-Hong

Lin, Chiu-Yue

Lin, Chun-Ling

Lin, Deyan

Lin, Jeng-Chyan Muti

Lin, Jing-Yuan

Lin, Jonqlan

Lin, Linhan

Lin, Qingyang

Lin, Sidney

Lin, Sijin

Lin, Tyrone $\mathrm{T}$.

Lin, Wenye

Lin, Yuan-Chung

Lin, Yu-Hsiu

Lin, Zhengyu

Lin, Zhibin

Linares, José Ignacio

Lind, Pedro G.

Lindholm, Andreas

Lineykin, Simon

Ling, Florence T.

Ling, Jiazhen

Ling, Meng

Ling, Steve

Liobikienè, Genovaitè

Liorni, Ilaria

Lipiński, Seweryn

Lips, Markus

Lisowski, Edward

Lister, Tedd E.

Litvić, Mladen

Litwin, Wojciech

Liu, Chunhua

Liu, Guodong

Liu, Hanchao

Liu, Huolong

Liu, Jau-Yang

Liu, Jianlin

Liu, Jinfeng

Liu, Lin

Liu, Meng-Kun (Jason)

Liu, Minglu

Liu, Pei 
Liu, Qi

Liu, Ren-Shiou

Liu, Shaoqing

Liu, Tianyu

Liu, Ting

Liu, Ting-Yu

Liu, Wan-Yu

Liu, Xiaobing

Liu, Yang

Liu, Yi-Hua

Liu, Ying

Liu, Yingyi

Liu, Yow-Chyi

Liu, Yucheng

Liu, Yuhao

Liu, Yu-Jen

Livanos, George

Liyanage, Wasantha

Lizana, Ricardo

Llopis, Rodrigo

Lo Storto, Corrado

Lo, Huang-Mu

Lo, Kin Hing

Lo, Wei-Shuo

Log, Torgrim

Lomonaco, Guglielmo

Lomonaco, Pedro

Long, John M.

Long, Philip

Longe, Omowunmi Mary

Longo, Michela

Longo, Sandro

Longo, Savino

López De Lacalle, Luis N.

Lopez Santos, Oswaldo

Lopez, Gartzen

López, José María

Lopez, Omar D.

López-Gutiérrez, José-Santos

Lopez-Iturri, Peio

López-Jiménez, P. Amparo

López-López, Daniel

López-Ochoa, Luis M.

Lorenz, Ralph D.

Lou, Fangyuan

Loures, Luis

Louwen, Atse

Lovas, Tamás

Love, Jenny

Lowry, Gordon

Lozano-Guerrero, Antonio José

Lozito, Gabriele Maria

Lu, Chia-Liang

$\mathrm{Lu}$, Fei

Lu, Jiang

Lu, John C.-C.

Lu, Junwei

Lu, Liqiang
Lu, Yan

Lucas Miralles, Manuel

Lucas, Alexandre

Lucas, Cândida

Lucchi, Elena

Lugauer, Steven

Lughofer, Edwin

Luís, Ricardo

Luisa, Alfieri

Luisetto, Igor

Łukasik, Rafał

Luna Martínez, Diego

Luna, Massimiliano

Lundmark, Sonja Tidblad

Luo, Jerry

Luo, Ming

Luo, Xiaobo

Lynch, Stephen

Lytras, Miltiadis D.

Lyu, Hailong

M. Shabestari, Parisa

Ma, Dan

Ma, Hui

$\mathrm{Ma}, \mathrm{Lu}$

Ma, Thi Thuong Huyen

Ma, Xiandong

Ma, Xiaoli

Maalouf, Chadi

Maat, Kees

Mabrouk, Mohamed Tahar

MacArthur, John

Maccioni, Marco

Mach, Pavel

Machado, Carolina

Macquart, Terence

Macucci, Massimo

Madadian, Edris

Madala, Srikanth

Madaleno, Mara

Madhusoodhanan, Sachin

Mady, Mohamed F.

Maestri, Matteo

Maga, Dusan

Magaril, Elena

Magasinski, Alexandre

Magazzino, Cosimo

Magistri, Loredana

Maglaras, Athanasios

Mago, Pedro J.

Magrini, Anna

Mahabadipour, Hamidreza

Mahanti, Aniket

Mahdavi, Mahboobe

Mahmoud, Alaa El Din

Mahmoudzadeh Andwari, Amin

Mahmud, Apel

Mahmud, Shohel

Mahvi, Allison 
Mahzari, Pedram

Maican, Edmond

Maicke, Brian

Maireles Torres, Pedro

Maisch, Tim

Maisonneuve, Jonathan

Maiz, Jon

Maj, Grzegorz

Majidi, Mehrdad

Majumdar, Payal

Mäkelä, Mikko

Maki, James

Mäki-Arvela, Päivi

Makkonen, Lasse

Makys, Pavol

Male, Frank

Malecha, Ziemowit

Malka, Dror

Malkowski, Piotr

Malpei, Francesca

Malvić, Tomislav

Malvoni, Maria

Malysz, Pawel

Manana, Mario

Manavalan, Balachandran

Mancilla-David, Fernando

Mancini, Francesco

Mancini, Marco

Mancini, Rock

Mancusi, Erasmo

Mandelli, Stefano

Mander, Ulo

Manfredini, Manfredo

Manfriani, Leonardo

Manganelli, Benedetto

Manganiello, Patrizio

Manhartsgruber, Bernhard

Manikas, Andrew S.

Manoj, Khandelwal

Manousakis, Nikolaos M.

Mansouri, Amir

Mantzaras, John

Manuella, Fabio Carmelo

Manzano-Agugliaro, Francisco

Manzone, Marco

Mao, Lei

Marafona, José Duarte

Marano-Marcolini, Alejandro

Maraveas, Chrysanthos

Marchesoni, Mario

Marchetti, Barbara

Marchi, Beatrice

Marchi, Michela

Marcin, Wardach

Marco, Mauri

Marcon, Petr

Marcu, Marius

Marek, Maciej
Margheritini, Lucia

Mari, Carlo

Mariano, Silvio

Marini, Martino

Marino, Concettina

Markides, Christos

Markondeya Raj, Pulugurtha

Markopoulos, Angelos P.

Marlair, Guy

Maroufmashat, Azadeh

Marques, António

Márquez, Fausto Pedro García

Marra, Francesco Saverio

Marsellos, Antonios

Martí, Pere

Marti-Herrero, Jaime

Martin Chivelet, Nuria

Martin, Claudia Fernandez

Martin, Jose Miguel

Martin, Russell

Martin, William

Martínez Torres, Javier

Martínez, Alberto González

Martinez, Patricio

Martinez, Sergio

Martinez, Wilmar

Martínez-Álvarez, Francisco

Martínez-Duart, J.M.

Martinez-Hernandez, Elias

Martinez-Laserna, Egoitz

Martínez-Lucas, Guillermo

Martinez-Molina, Antonio

Martinez-Ramos, José L.

Martinez-Triguero, Joaquin

Martin-Gomez, Cesar

Martín-Gómez, César

Martini, Alberto

Martín-Martínez, Francisco María

Martinopoulos, Georgios

Martín-Rubio, Irene

Martín-Yerga, Daniel

Martowicz, Adam

Marucci, Alvaro

Marugán, Alberto Pliego

Maryska, Milos

Marzband, Mousa

Marzbani, Hormoz

Masayuki, Anyoji

Masek, Vlastimil

Masini, Barbara Mavi

Maslennikov, Slava

Masnicki, Romuald

Massaglia, Giulia

Masselli, Claudia

Massidda, Luca

Massier, Tobias

Massimo, Rundo

Mastrucci, Alessio 
Masutani, Stephen

Mata, Érika

Mata, Maria Del Carmen

Matagne, Ernest

Matas, José

Mateo-Lázaro, Jesús

Mateos, Esperanza

Mateus, Nuno

Mathieu-Potvin, François

Matias, João C. O.

Matias, Sessarego

Matko, Vojko

Matos, Joao Nuno

Matsakas, Leonidas

Matsuda, Yoshitaka

Matsumoto, Ken'ichi

Matsumoto, Satoshi

Matteo, Savaresi Sergio

Mauree, Dasaraden

Mauri, Roberto

Mauriello, Francesco

Mauro, Gerardo Maria

Mauro, Stefano

Mavromatakis, Fotis

Mavropoulos, Georgios

Maximiano, Marisa Da Silva

Mayeed, Mohammed Sajjad

Mayer, Audrey

Mazidi, Peyman

Mazzeo, Domenico

Mazzoccoli, Michela

McDaniel, Patrick

McEnally, Charles

McGowan, J.G.

McGrattan, Kevin B.

Medina Quesada, Ángeles

Medina, Agustin Valera

Medina-Padrón, José F.

Medina-Padron, Jose Fernando

Meegahapola, Lasantha G

Meerman, Hans

Megalos, Mark A.

Mehdi, Rahmani-andebili

Mehdipour, Iman

Mehmood, Khawaja Khalid

Mehrabadi, Mohammad Allahdadi

Mehran, Kamyar

Mehrdad, Salehi

Mehrkesh, Amirhossein

Meiss, Alberto

Melcher, Andreas

Meléndez, Joaquim

Memon, Saim

Mendes, André

Mendes, Goncalo

Mendes, Jérôme

Mendes, Mário José Gonçalves Cavaco

Mendoza, Daniel
Menegaki, Angeliki

Meneghetti, Antonella

Meneghetti, Giovanni

Menéndez, Miguel

Meneses Rioseco, Ernesto

Meng, Jing

Meng, Wenchao

Meng, Xiangbo

Menoufi, Karim

Merabet, Adel

Merchan-Merchan, Wilson

Mercorelli, Paolo

Merenda, Massimo

Merk, Bruno

Merkel, Broder

Merola, Simona Silvia

Messineo, Antonio

Meyer, Ina

Meyer, Richard T.

Meyer-Kohlstock, Daniel

Mi, Zhifu

Miao, Guoxing

Micallef, Daniel

Miccio, Francesco

Michailides, Constantine

Michailidis, Iakovos

Michel, Jean-Bernard

Michela, Vellini

Michiorri, Andrea

Midttømme, Kirsti

Mihalic, Rafael

Mihaylov, Mihail

Mihelic, Matevz

Mihet, Lucian

Mihet-Popa, Lucian

Mikayilov, Jeyhun I

Mikropoulos, Pantelis N.

Milewski, Jarosław

Millar, John

Milone, Candida

Milovanovic, Bojan

Min, Baehyun

Min, Jihoon

Minakshi, Manickam

Minami, Yuki

Mindykowski, Janusz

Minea, Alina Adriana

Minnaert, Ben

Miranda, Vladimiro

Mishra, Aashwin

Mishra, Aditya Kumar

Mishra, Rajesh

Mishra, Rakesh

Mistrick, Richard G.

Misyura, Sergey Ya

Mitani, Tomohiko

Miyatake, Masafumi

Miyazaki, Takahiko 
Mizielińska, Małgorzata

Mizsei, János

Mkandawire, Martin

Mlejnek, Pavel

Młyński, Dariusz

Mo, Kun

Mobley, Justin

Mochizuki, Hiroyasu

Mocz, Philip

Modepalli, Kumar

Modoni, Gianfranco

Moeller, Devin

Moghaddami, Masood

Mohamed, Ryadi

Mohammadi, Javad

Mohammadzadeh, Omid

Mohammed, Hussein A.

Mohd Alsofyani, Ibrahim

Mohsenzadeh, Amin

Moiz, Ahmed Abdul

Mokhtari, Mehdi

Moldovan, Macedon

Molin, Eric

Molina-Aiz, Francisco

Molina-Garcia, Angel

Molinas, Marta

Moliner, Cristina

Molino, Antonio

Moll, Henri C.

Molnar-Matei, Florin

Momete, Daniela

Moncada, Stefano

Mondal, Abrez

Mondéjar, José Antonio Rodríguez

Mondejar, Maria E.

Monforti-Ferrario, Fabio

Mongiardo, Mauro

Monino, Antonio

Monsalve-Serrano, Javier

Montalvo, Carlos

Montecucco, Andrea

Monteiro, Eliseu

Monteiro, Joaquim

Monteiro, Vítor

Monteiro, Vítor Duarte Fernandes

Montes, Hector

Montilla-DJesus, Miguel E.

Moon, Seung Eon

Moonem, M A

Moonem, M.A.

Moore, Philip

Moore, Trivess

Moradi, Bahareh

Morán Álvarez, Antonio

Moran, Daniel

Moravek, Jan

Morávková, Zuzana

Morea, Donato
Moreno, Valentín Molina

Moreno-Atanasio, Roberto

Moreno-Muñoz, Antonio

Moretti, Elisa

Morey, R. Vance

Morganti, Michele

Morganti, Pierfrancesco

Moriarty, Patrick

Morineau, Thierry

Morkvenaite-Vilkonciene, Inga

Moron, Carlos

Morone, Piergiuseppe

Moroni, Davide

Morosi, Simone

Morozyuk, Tatjana

Morrone, Pietropaolo

Morshed, Khandakar Niaz

Morshed, Mohammad Javad

Mortazavi, Amir

Mortazavi, Bohayra

Mortazavian, Shahed

Moshou, Dimitrios

Moshrefi-Torbati, Mohamed

Moskalik, Tadeusz

Mota-Babiloni, Adrián

Motalleb, Mahdi

Motra, Hem Bahadur

Mottola, Fabio

Moujaes, Samir

Mount, Timothy

Mousa, Wael

Mousavi, S. Mostafa

Moutinho, Victor

Mowry, Greg

Mubenga, Ngalula

Muc, Aleksander

Mudunuru, Maruti Kumar

Mueller, David

Muhammad-Sukki, Firdaus

Mujica, Gabriel

Mulas, Marco

Müller, Julian Marius

Müller, Karsten

Müller-Buschbaum, Peter

Mullins, Oliver C.

Mummolo, Giovanni

Münch-Alligné, Cécile

Muniak, Damian Piotr

Munir, Tariq

Muntean, Sebastian

Munteanu, Calin

Munters, Wim

Murai, Yuichi

Muro Jr., Aldo

Murphy, Fionnuala

Murphy, Michael D.

Murray, John

Muscio, Alberto 
Musilek, Petr

Musmarra, Dino

Muster, Tim

Mustoe, George E.

Muszyński, Tomasz

Mysliwiec, Tami

Mystkowski, Arkadiusz

Nad, Alona

Nadais, Helena

Naderi, Seyed Behzad

Naderian, Ali

Nadolny, Zbigniew

Næss, Erling

Nagarajan, Magesh

Nagendra, Ganesh

Nagy, Endre

Naidu, Desineni Subbaram

Nájera, Jorge

Nakata, Toshihiko

Nalepa, Jakub

Nalim, M. Razi

Nam, Yujin

Nambiar, Anup

Nan, Fany

Nanaki, Evanthia

Nandi, Tarak Nath

Nankervis, Alan

Nankoo, Daniel

Naraghi, Mohammad

Narciso, Javier

Nardecchia, Fabio

Narimani, Mohammad Rasoul

Naseer, Noman

Nasri, Bayan

Nastasi, Benedetto

Nastro, Rosa Anna

Natan, Benveniste

Natarajan, Anand

Natarajan, Mahesh

Naumann, Andre

Navalon, Sergio

Nawaz, Syed Junaid

Nazaripouya, Hamidreza

Nazir, Shareq Mohd

Nchanji, Eileen Bogweh

Neagoe, Mircea

Neelameggham, Neale R

Neely, Jason C.

Nehaoua, Lamri

Nejati, Siamak

Nelatury, Sudarshan

Nelson, Tim

Nemes, Ciprian

Nemś, Artur

Nemś, Magdalena

Nepal, Rabindra

Neretti, Gabriele

Neri, Davide
Neuberger, Pavel

Neuman, B. Clifford

Neumann, Holger

Neumann, Mathias

Neves, Luís Pires

Newaz, Mohammad

Nezamoddini, Nasim

Nezhad, Mohaddeseh Mousavi

$\mathrm{Ng}$, Bing Feng

$\mathrm{Ng}$, Derrick Wing Kwan

Nguyen, Binh P.

Nguyen, Duy-Dinh

Nguyen, Gia Minh Thao

Nguyen, Ngoc Tu

Nguyen, Thi Bich Ngoc

Nguyen, Tu (Ryan)

Nguyen, Van Hoa

Nguyen, Xuan Bac

Nguyen-Van, Triet

$\mathrm{Ni}$, Kai

Nicodemo, Gianfranco

Nicolas-Apruzzese, Joan

Nicolini, Andrea

Nie, Mengyan

Nie, Zhenggang

Niedbalski, Zbigniew

Nieken, Ulrich

Nielsen, Per Sieverts

Nielsen, Peter

Nielsen, Søren Nors

Nielsen, Vilhjálmur

Nieminen, Kaarlo

Nieße, Astrid

Niguchi, Noboru

Nijhuis, Michiel

Nikas, George K.

Nikitas, Alexandros

Nikolaidis, Th.N.

Nikolaos, Kiratzis

Nikolaou, Ioannis

Nikolaou, Michael

Nikolic, Dragan

Nikolic, Mijo

Nikolovski, Srete

Nikparto, Ali

Nishitateno, Shuhei

Niu, Shuangxia

Nivedita, Nivedita

Nizetic, Sandro

Nižetić, Sandro

Nobrega, Rodolfo

Nocente, Alessandro

Nocera, Francesco

Noetinger, Benoît

Nofuentes, Gustavo

Noga, Marcin

Noghanian, Sima

Nogueira, José-Ignacio 
Nogueira, Teresa

Nojabaei, Bahareh

Nones, Michael

Nord, Natasa

Norici, Alessandra

Norrlund, Per

Notingher, Petru

Notton, Gilles

Nour, Mutasim

Nouri Gharahassanlou, Amin

Noussan, Michel

Novák, Tamás

Novas, Nuria

Nowak, Andrzej

Ntanos, Stamatios

Ntougias, Spyridon

Nuchkrua, Thanana

Nunes, Leonel

Nuñez, Neftali

Nuñez, Ruben

Obeng-Gyasi, Emmanuel

Obidziński, Sławomir

Obonyo, Esther

Obonyo, Esther A.

Obracaj, Dariusz

O'Brien, Paul

Ochowiak, Marek

Oclon, Pawel

Odeh, Saad

O'Donnell, Terence

Odriozola, José A.

Oggeri, Claudio

Ogonowski, Szymon

Ogorek, Rafal

O'Hagan, Anne Marie

Ohba, Ryohji

Ohenoja, Markku

Ohnuma, Takumi

Ohsawa, Teruo

Ohtake, Hideaki

Oikonomou, Konstantinos

Okamoto, Satoru

Okarma, Krzysztof

Okumura, Susumu

Olalla, Carlos

Olchowka, Jacob

Oliveira, José António Vasconcelos

Oliveira, Rafael

Oliveira, Santiago

Oliver, Albert

Olmeda, Pablo

Olmedillas Blanco, Blanca

Omitaomu, Olufemi A.

Omranian, Ehsan

Oncioiu, Ionica

Onder, Christopher

Ondrejka Harbulakova, Vlasta

Onea, Florin
Oneto, Luca

Onica, Ilie

Onishi, Viviani

Opel, Oliver

Oprea, Razvan Andrei

Oprea, Simona

Orejon, Daniel

Orikawa, Koji

Orlandi, Antonio

Orrego, David

Ortega, José Marcos

Ortiz, Alfredo

Orts-Grau, Salvador

Orynycz, Olga

Osaba, Eneko

Osatiashtiani, Amin

Osmond, Paul

Ostadhassan, Mehdi

Österreicher, Doris

Ostoja-Starzewski, Martin

Otsuka, Akihiro

Otsuki, Akira

Ottonelli, Massimo

Ou, Ting-Chia

Ou, Xunmin

Ouchida, Yasunori

Oureilidis, Konstantinos

Ouro, Pablo

Outbib, Rachid

Overbye, Thomas J.

Ożadowicz, Andrzej

Ozarisoy, Bertug

Ozawa, Akito

Pablo, Santiago De

Pablo-Romero, María Del P.

Paccagnini, Alessia

Padilla-Marcos, Miguel Ángel

Padilla-Rivera, Alejandro

Paeglitis, Ainars

Paez Ayuso, Javier

Pagliarini, Giorgio

Pagnotta, Daniele Proietti

Pagonis, Dimitrios-Nikolaos

Painter, Roger

Paiva, Sara

Paixão Cansado, Isabel Pestana

Pakka, Vijayanarasimha Hindupur

Pal, Pinaki

Paladino, Ombretta

Palella, Boris Igor

Palme, Massimo

Palmer, Diane

Palmieri, Marco

Palomares-Salas, José Carlos

Palsson, Halldor

Paluszczyszyn, Daniel

Pan, Haozhi

Pan, Huilin 
Pan, Kai

Pan, Qin

Pan, Seon

Pan, Shu-Yuan

Pan, Wei-Ping

Panagiotidis, Theodoros

Panapakidis, Ioannis P.

Panaras, Giorgos

Panchal, Satyam

Panepinto, Deborah

Pani, Shantanu Kumar

Panico, Antonio

Panjwani, Deep

Panomsuwan, Gasidit

Pant, Deepak

Pantaleo, Antonio

Paolini, Riccardo

Papadimitriou, Christina

Papadopoulos, Agis M.

Papadopoulos, Theofilos

Papaelias, Mayorkinos

Papageorgas, Panagiotis

Papagiannakis, Roussos G.

Papanikolaou, Nick

Papathanassis, Alexis

Papista, Eleni

Pappalardo, Carmine Maria

Papurello, Davide

Parajuli, Ranjan

Pardo, Miguel

Parente, Alessandro

Pareschi, Fabio

Parisi, Alfio

Parithi, Ilam

Park, Byung-Wook

Park, Changhyup

Park, Daeryong

Park, Hyeongon

Park, Ji-Won

Park, Jonghyun

Park, Joong Yull

Park, Joontaek

Park, Kenneth S

Park, Kyung-Soon

Park, Sang Kil

Park, Sangki

Park, Sehyun

Park, Sungwook

Park, Sunho

Park, You-Jin

Park, Young-Kwon

Parnell, Andrew J.

Parra, Lorena

Parsegov, Sergei

Pascual, Julio

Pascual-Muñoz, Pablo

Pasetti, Marco

Pastore, Luisa
Patchigolla, Kumar

Patel, Sarosh

Paterson, Nigel

Patil, Abhay

Patil, Madhav

Patriarca, Riccardo

Patruno, Luca

Patsios, Sotiris I.

Patton, Ron J.

Pau, Giovanni

Paul, Anand

Paul, Sanjoy

Paul, Titan C.

Paulech, Juraj

Paulis, Francesco De

Paun, Dragos

Pauss, Andre

Pavan, Paolo

Pavković, Danijel

Pavle, Jakovac

Paweł, Pławiak

Pawlak-Kruczek, Halina

Pawlowski, Andrzej

Pawlus, Witold

Payá, Luis

Peace, Alexander

Peampermpool, Rakpong

Pearce, Julie

Peças Lopes, João

Pecorella, Tommaso

Pecori, Riccardo

Pedreiras, Paulo

Peer, Akshit

Peeters, Floran

Pehlken, Alexandra

Pei, Peng

Peimankar, Abdolrahman

Peinke, Joachim

Peinl, René

Peischl, Bernhard

Pellegrini, Marco

Pelusi, Danilo

Peña García, Antonio

Penalba, Markel

Peng, Jun

Peng, Teng

Peng, Wei

Pennestrì, Ettore

Pensieri, Sara

Peppas, Georgios

Perazzo, Antonio

Perea Moreno, Alberto Jesus

Pereira, Adelino J. C.

Pereira, Amaro Olimpio

Pereira, Lucas

Pereira, Paulo Rogerio

Perera, Sudantha

Peres, David 
Pérez Sánchez, Modesto

Perez, Hector

Pérez, Noelia Uribe

Pérez-Collazo, Carlos

Pérez-García, Julián

Perikos, Isidoros

Perin, Giorgio

Perini, Roberto

Peris, Alfredo

Pernía, Alberto M.

Pernigotto, Giovanni

Pesyridis, Apostolos

Peters, Ralf

Peterson, Rorik

Petrakis, Evangelos

Petrella, Andrea

Petreus, Dorin

Petrone, Giovanni

Petrosanu, Dana-Mihaela

Petrovic, Stefan

Petru, Livinti

Pettersen, Johan Berg

Peyghami, Saeed

Pezzuolo, Andrea

Pezzutto, Simon

Pfost, Heiner

Pfost, Martin

Pham, Phuong

Phillippa, Bronson

Phillips, Stephen C.

Phu Nguyen, Vinh

Piana, Giulia

Piasecki, Krzysztof

Piatek, Zygmunt

Picard, Damien

Picchio, Rodolfo

Pickl, Stefan

Piechna, Janusz

Pielech-Przybylska, Katarzyna

Pieper, Jeff

Pietrowicz, Sławomir

Pietrusiak, Damian

Pietrzyk, Piotr

Pillai, Gobind Gopalakrishna

Pilloni, Virginia

Pin, Jean-Mathieu

Pina Varas, Perla

Pinares, Gustavo

Pindado, Santiago

Pindozzi, Stefania

Pinheiro, Mario

Pinho, Pedro

Pintauro, Peter N.

Pinto, José Gabriel Oliveira

Pinto, Sonia F.

Pinto, Sónia F.

Pinto, Tiago

Pinzi, Sara
Pio, Gianvito

Piot-Lepetit, Isabelle

Piperidis, Savvas

Piqueras, Pedro

Piraghaj, Sareh Fotuhi

Pires Manso, Jose Ramos

Pirinen, Pekka

Pirjan, Alexandru

Pirraglia, Adrian

Pisano, Giuditta

Pisello, Anna

Pislaru-Danescu, Lucian

Pistikopoulos, Efstratios

Pisupati, Sarma V.

Pittermann, Martin

Pitto, Andrea

Piwowar, Arkadiusz

Plácido-Escobar, Jersson E.

Platero, Carlos A.

Pławiak, Paweł

Plesca, Adrian

Plesca, Adrian Traian

Pode, Ramchandra

Podporkin, Georgij

Polajžer, Boštjan

Polanský, Radek

Pollitt, Michael

Polverino, Pierpaolo

Pompili, Massimo

Poncela, Javier

Poncet, Sébastien

Ponta, Linda

Pontelli, Enrico

Pooranian, Zahra

Poós, Tibor

Poozesh, Sadegh

Popa, Bogdan

Popa, Gabriel Nicolae

Popescu, Mihaela

Popli, Nipun

Popp, Denny

Popp, József

Poredoš, Primož

Porru, Mario

Porteous, Colin

Porumb, Radu

Postigo Marcos, Fernando E.

Postnikov, Ivan

Potapenko, Dmitriy

Poudel, Jeeban

Poulios, Ioannis G.

Poupin, Christophe

Pourakbari Kasmaei, Mahdi

Pouresmaeil, Edris

Pourmousavi Kani, Seyyed Ali

Pourrahimi, Amir Masoud

Poursadegh, Farzad

Pous, Narcis 
Povinelli, Richard J.

Povstenko, Yuriy

Powell, Helen

Powell, Jonathan P.

Powell, Kody

Poza Lobo, Javier

Pozo, Francesc

Praca, Isabel

Pradhan, Nirakar

Prado, Raquel

Prakht, Vladimir

Praktiknjo, Aaron

Prasad, Abhnil

Prasad, Gautham

Prasanth, Venugopal

Precup, Radu-Emil

Pręgowska, Agnieszka

Preißinger, Markus

Premanode, Bhusana

Prendes-Gero, Maria Belen

Prentkovskis, Olegas

Preve, Nikolaos P.

Pricop, Emil

Priede, Janis

Prieto, Jesús-Ignacio

Prlić Kardum, Jasna

Procek, Marcin

Procopio, Renato

Proenca, Sara

Pröll, Tobias

Proto, Andrea Rosario

Prusek, Stanisław

Przyborski, Marek

Przybylek, Piotr

Psomopoulos, Constantinos S.

$\mathrm{Pu}$, Cong

$\mathrm{Pu}$, Hui

$\mathrm{Pu}$, Jaan $\mathrm{H}$

Puddu, Pierpaolo

Puente, Santiago

Pugi, Luca

Pugliese, Francesco

Pujol Nadal, Ramon

Pujol, Toni

Pullen, Keith Robert

Pyrgioti, Eleftheria

Qi, Zhaoxiang

Qian, Hua

Qiang, Zhe

Qiu, Guanglei

Qiu, Songgang

Qiu, Xin

Qiu, Yonghe

Quaranta, Emanuele

Quattrini Li, Alberto

Quintana Barcia, Pablo

Quirion, Philippe

Rabczuk, Timon
Rabie, Khaled

Rabinovici, Raul

Radac, Mircea-Bogdan

Radhakrishnan, Chandra

Radica, Gojmir

Radu, Laura-Diana

Radulovic, Jovana

Radwan, Amr

Rafael, Silviano

Rafajdus, Pavol

Ragab, Tarek

Ragauskas, Arthur J.

Ragauskas, Arthur Jonas

Rago, Laura

Rahbari Asr, Navid

Raheem, Adeeba Abdul

Rahman, Khondokar Mizanur

Rahman, Md Shihanur

Rahman, Md. Mahbubur

Rahman, Md. Mofijur

Rahman, S.M. Ashrafur

Rahman, Ziaur

Rahmani, Omeid

Rahmat, Ellahi

Rahnema, Hamid

Rainey, Thomas

Rajchowski, Piotr

Rakopoulos, Constantine D.

Ramakumar, Rama

Ramandi, Hamed Lamei

Ramírez, Martín

Ramis Ferrer, Borja

Ramis, Gianguido

Ramos Cabal, Alba

Ramos Paja, Carlos Andres

Ramos, Célia M. Q.

Ramos, Helena

Ramos, João António Esteves

Ramos-Hernanz, Josean

Ramos-Suárez, Juan Luis

Rana, Dipak

Rana, Zeeshan

Ranaee, Ehsan

Randhir, Kelvin

Rangel, Victor

Rangel-Hernandez, Victor

Raniszewski, Grzegorz

Ranjkesh, Amid

Rantuch, Peter

Rao, Agnè

Rao, Ajith

Räsänen, Timo

Rasheduzzaman, Md

Rasheed, Eziaku

Rashid, Mudassir

Rashidi, Mohammad Mehdi

Rassõlkin, Anton

Rath, Jagat Jyoti 
Rathnaweera, Tharaka

Ratner, Albert

Rattner, Alexander S.

Rau, Matthew J.

Rauch, Reinhard

Rauh, Andreas

Rauwoens, Pieter

Ravaghi, Zohreh

Ravelli, Davide

Ravikumar, Kumar

Razeghi, Ghazal

Razik, Hubert

Real-Calvo, Rafael

Reatti, Alberto

Reddy, S. Surender

Redondo, Jose Manuel

Redondo-Iglesias, Eduardo

Rees, D. Andrew S.

Regueiro, Araceli

Reinap, Avo

Reinders, Angele

Reinmöller, Markus

Rekioua, Djamila

Remaggi, Luca

Ren, Hao

Ren, Jingzheng

Ren, $Y \mathrm{i}$

Ren, Zhen

Rendueles, Manuel

Renevey, Philippe

Rengasamy, Karthikeyan

Renna, Paolo

Renzi, Massimiliano

Reverberi, Andrea P.

Revollar, Silvana

Rey Martinez, Francisco Javier

Reyes-Belmonte, Miguel A.

Reyes-Labarta, J. A.

Reza, Mohammad Toufiq

Rezaei-Gomari, Sina

Rezania, Shahabaldin

Rhee, Kyu-Nam

Rhee, Sang-Bong

Rhi, Seok-Ho

Riaud, Antoine

Riba, Jordi-Roger

Ribeiro, Luís Frölén

Ribeiro-Soriano, Domingo

Ribič, Janez

Ricci, Alessio

Ricci, Stefano

Ricciardi, Vincenzo

Riccio, Giuseppe

Ricco, Mattia

Richardson, Walter

Richelli, Anna

Richter, Andreas

Richtera, Lukáš
Ries, Robert J.

Riganti Fulginei, Francesco

Rigby, Sam

Rikos, Evangelos

Rimkus, Alfredas

Rinaldi, Fabio

Rinaldi, Stefano

Ringwood, John

Riordan, Daniel

Ripka, Pavel

Ripollés, Jordi

Rishpon, Judith

Ristic, Michael

Ritter, Matthias

Riva Sanseverino, Eleonora

Rizzardi, Alessandra

Rizzo, Fabio

Rizzo, Santi Agatino

Rizzoli, Gabriele

Robert, Tanguy

Roberto Sebastiano, Faranda

Roberto, Corizzo

Roberts, Nicholas

Robertson, Amy

Robertson, Bryson

Robison, Andrew

Rocco, Matteo Vincenzo

Rocha, Alfredo

Rock, Michael

Rodenas-Herráiz, David

Rodger, James A.

Rodgers, Mark

Rodic, Miran

Rodrigues, Celestino

Rodrigues, João

Rodrigues, José Miguel

Rodrigues, Nelson E. V.

Rodriguez Diaz, Enrique

Rodríguez, Fco

Rodríguez, Francisco J.

Rodríguez, Jaime

Rodriguez, Sergio

Rodríguez-Martín, Manuel

Rodríguez-Soalleiro, Roque

Rodriguez-Ubinas, Edwin

Rodziewicz, Joanna

Roehrich, Jens

Rogers, Eric

Rogowski, Krzysztof

Rohacs, Jozsef

Roldán Pérez, Javier

Rolland, Sam A.

Romagnoni, Percarlo

Romagnoni, Piercarlo

Roman, Raul-Cristian

Román, Rocio

Romar, Henrik

Römer, Ulrich 
Romero, Isabel

Rona, Aldo

Roncero-Sánchez, Pedro

Ronen, Avner

Roque, Luís A. C.

Rosa, Flavio

Rosa, Rui Namorado

Rosales-Asensio, Enrique

Roscia, Mariacristina

Roselli, Carlo

Ros-McDonnell, Lorenzo

Rossella, Francesco

Rossi, Federico

Rossi, Giuliana

Rostami, Ali Bakhshandeh

Rottengruber, Hermann

Rottondi, Cristina

Roubík, Hynek

Rougier, Esteban

Roumpos, Christos

Rovey, Joshua L.

Rovira, Antonio

Roy Chowdhury, Bikram

Roy, Abhishek

Roy, Jinia

Roy, Spandan

Roy, Sukanta

Rozga, Pawel

$\mathrm{Ru}$, Pengkai

Ruan, Jiageng

Rubino, Guido

Rubio-Bellido, Carlos

Ruble, Isabella

Ruda González, Albert

Ruderman, Alexander

Rudez, Urban

Ruffino, Barbara

Rugnini, Lorenza

Rui, Zhenhua

Ruiz, Fernando

Ruiz, Jordi-Roger Riba

Ruiz, M. Pilar

Ruiz-Femenia, Ruben

Ruiz-García, Alejandro

Ruiz-Rodriguez, Francisco Javier

Rupprich, Marco

Rus Casas, Catalina

Rushforth, Richard

Rusin, Andrzej

Russo, Carlo

Rusu, Eugen

Rusu, Liliana

Ruz, Francisco

Rybach, Ladislaus

Rybarczyk, Piotr

Rychlik, Igor

Rydosz, Artur

Ryu, Duchwan
Ryu, Jehwang

Ryu, Keun Ho

Rząsa, Janina

Saad, Walid

Saafi, Mohamed

Saber, Esmail M.

Sabharwal, Chaman

Sabzehgar, Reza

Saccani, Cesare

Sadaka, Sammy

Sadi, Mohammad Ashraf Hossain

Sadiki, Amsini

Sadorsky, Perry

Sadrzadeh, Mohtada

Saeed, Salimzadeh

Sáenz, Jon

Safa, Meer

Safacas, Athanasios

Safaei, Mohammad Reza

Šafarič, Riko

Safdarnejad, Seyed Mostafa

Saffari Pour, Mohsen

Safizadeh, M. Reza

Sager, Jalel

Saha, Bidyut Baran

Saha, Sushanta Kumar

Sahoo, Kamalakanta

Sainsbury, Bre-Anne

Saizarbitoria, Iñaki Heras

Sajid, Muhammad

Sajjadian, Masoud

Salamone, Francesco

Salas, Vicente

Salata, Ferdinando

Salcedo-Sanz, Sancho

Salè, Silvia Omodeo

Salehi, Rasoul

Salehi, Saeed

Salerno, Valerio Mario

Salimifard, Parichehr

Salmachi, Alireza

Saloux, Etienne

Salvadori, Giacomo

Salvadori, Simone

Salvalai, Graziano

Salvati, Luca

Salvini, Coriolano

Salvo, Francesca

Salzano, Ernesto

Samadani, Ehsan

Samaras, Achilleas

Samaras, Ioannis

Samborski, Sylwester

Samuel, Edmund

Samuele, Lilliu

Sánchez De La Flor, Francisco

Sánchez, Antonio

Sánchez, D. 
Sanchez, Manuel

Sánchez-Fernández, José Ángel

Sánchez-Ostiz Gutiérrez, Ana

Sanchis, Javier

Sandberg, Thomas

Sandborn, Peter

Sander, Regina

Sandou, Guillaume

Sandoval, Diego R.

Sandrolini, Leonardo

Sanduleac, Mihai

Sangiorgio, Matteo

Sanjari, Mohammad Javad

Sanjou, Michio

Sannan, Sigurd

Sanphuang, Varittha

Sansom, Christopher

Santafé, Miguel Redón

Santamaría-Peña, Jacinto

Santana, Jose

Santander-Jiménez, Sergio

Santangelo, Paolo E.

Santiago, Isabel

Santilano, Alessandro

Santini, Ezio

Santoro, Francesco

Santos, Paulo

Sanwar Hosen, A. S. M.

Sanz Bobi, Miguel Angel

Sanz-Bobi, Miguel A.

Saponara, Sergio

Sarafidis, Yannis

Sarjaš, Andrej

Sarkar, Biswajit

Sarkar, Mohan

Sarrias-Mena, Raúl

Sarshar, Marjan

Sasaki, Ken

Sathyan, Shelas

Sauhats, Antans

Savard, Christophe

Savitski, Dzmitry

Saxen, Henrik

Saydam, Serkan

Sayed, Khairy

Scandurra, Giuseppe

Scappaticci, Lorenzo

Schaefer, Hans-Eckhardt

Schäfer, Benjamin

Scheffe, Jonathan

Schegner, Peter

Scheidl, Rudolf

Schenone, Corrado

Schiemann, Martin

Schiffer, Jürgen

Schirone, Luigi

Schissler, Andrew

Schito, Eva
Schlosser, Viktor

Schmidt Davidsen, Rasmus

Schmidt, Uwe

Schmitz, Sven

Schneider, Petra

Schoenwald, David

Schormans, John

Schröder, Uwe

Schueller, Michael

Schultz, Michael

Schumacher, Sandra

Schuttrumpf, Holger

Schwartz, David Eric

Schwarz, Philip

Schweiss, Ruediger

Schwerin, Hagen

Sciubba, Enrico

Scoccia, Rossano

Scogings, Chris

Scribano, Vittorio

Scrivener, Colin

Scurrell, Mike

Sechilariu, Manuela

Se,dziwy, Adam

Segurado, Raquel

Seifitokaldani, Ali

Sellami, Nazmi

Selle, Laurent

Selosse, Sandrine

Selvaprabhu, Poongundran

Semaltianos, Nikolaos G.

Semkov, Krum

Senabre, Carolina

Senatore, Luigi

Sendin, Alberto

Senetakis, Konstantinos

Senjyu, Tomonobu

Seo, Hun-Chul

Seo, Youngmin

Sepasi, Saeed

Sequeira, César

Serati, Mehdi

Seritan, George

Serna, Sergio

Serpi, Alessandro

Serra, Sylvain

Serrano González, Javier

Serrano, Jose

Serrano, Luis

Servadio, Pieranna

Sesana, Marta Maria

Sescu, Adrian

Seshagiri, Sridhar

Setti, Dinesh

Sfarra, Stefano

Sferra, Adriana

Sfetsos, Athanasios

Sgroi, Mauro Francesco 
Sha, Ang

Shabgard, Hamidreza

Shadloo, Mostafa

Shadloo, Mostafa Safdari

Shafiee, Mahmood

Shafiei, Ali

Shafiel, Ali

Shafiq, Muhammad

Shafiullah, GM

Shah, Shahil

Shahparasti, Mahdi

Shahrokhi, Ava

Shaker, Hamid Reza

Shala, Ahmet

Shalaby, Mohamed

Shan, Ming

Sharifi, Ayyoob

Sharma, Ashish

Sharma, Yogesh

Shaw, Dein

She, Xiaohui

Sheikh, Shaya

Shekhar, Aditya

Shen, Chih-Lung

Shen, Weixiang

Sheng, Wanan

Sher, Farooq

Sherburn, Jesse A.

Sheremet, Mikhail

Sheu, Jeng-Shin

Shewarega, Fekadu

Shi, Jing

Shi, Suan

Shi, Wenbo

Shi, Xiaoyang

Shi, Xunpeng

Shi, Yang

Shi, Ye

Shibahara, Makoto

Shieh, Hsin-Jang

Shih, Ching-Long

Shimada, Koji

Shin, Chee Burm

Shin, Ju-young

Shin, Myunghun

Shin, Seoyong

Shinya, Norio

Shishkin, Andrei

Shojafar, Mohammad

Shor, Roman

Shrirao, Anil B.

Shroff, Sonell

Shu, Kesheng

Shukla, Sudheesh K.

Shvartsman, Vladimir

Sibilski, Krzysztof

Sicklinger, Stefan

Siebenhofer, Matthäus
Siegel, Nathan P.

Siegel, Stefan

Siemers, Werner

Sierpiński, Grzegorz

Sikorski, Wojciech

Siksnelyte, Indre

Silva, Fernando

Silva, J. Fernando A.

Silva, Patrícia Pereira Da

Silva, Sandra

Silva, Valter

Silva-Castro, Jhon

Silvestre, Miguel

Sim, Seung-Gyu (Andrew)

Simani, Silvio

Simeonov, Ivan

Simic, Milan

Simões, Teresa

Simone, Silvia De

Simonetti, Irene

Simpson, Genevieve

Singh, Amit Kumar

Singh, Dhananjay

Singh, Madhusudan

Singh, Rajveer

Singh, Robin

Singh, Shiwani

Singh, Shobhana

Singla, Mithun

Sinha, Nityanand

Sinha, Yashwant

Sioshansi, Ramteen

Sirghi, Lucel

Sisworahardjo, Nur

Sivasankaran, Harish

Sivasubramanian, Jayahar

Sjeric, Momir

Skaf, Dorothy W.

Skiba, Marta

Skoczylas, Norbert

Skoulou, Vassiliki K.

Škraba, Andrej

Skrzypkowski, Krzysztof

Sladic, Sasa

Slama, Mohammed El Amine

Slaski, Grzegorz

Ślefarski, Rafał

Sleszynski, Wojciech

Sleutels, Tom

Śliwiński, Paweł

Sližytè, Danutè

Slowakiewicz, Miroslaw

Ślusarczyk, Beata

Smalley, Craig

Smarandache, Florentin

Smarzewski, Piotr

Smethurst, Philip

Smirniotis, Panagiotis G. 
Smirnov, Alexander

Smith, Joseph D.

Smith, Marilyn

Smith, Thomas J.

Smoliński, Adam

Smyth, Beatrice

Snape, Richard

Soares, Joao

Soava, Georgeta

Sobus, Jan

Sockeel, Nicolas

Söffker, Dirk

Sofronov, Georgy

Sohoulande, Dagbegnon Clement

Sokoloff, Dmitry

Soldo, Vladimir

Soleimani, Majid

Soliman, Abdel-Hamid Ali

Solomon, Barry

Solomon, Yair

Solouk, Ali

Soltani, Amir

Soltani, Madjid

Soltero, Víctor M.

Soma, Gian Giuseppe

Somakettarin, Natthawuth

Soman, Ruturaj

Somoza, Luís

Son, Changmin

Son, Ki-Young

Son, Moon

Song, Yipeng

Song, Yongze

Song, Ziyou

Sonnino, Giorgio

Sopegno, Alessandro

Soria-Verdugo, Antonio

Sorniotti, Aldo

Soroudi, Alireza

Sossan, Fabrizio

Soukissian, Takvor

Sousa Figueira Barata, Filipe André De

Sousa, João C.

Sousa, Paulo Morgado

Sousa, Rita

Southall, Ryan

Sowale, Ayodeji

Sowizdzal, Anna

Spagnuolo, Giovanni

Spiess, Antje

Spina, Roberto

Spinazze, Andrea

Sportelli, Maria Chiara

Sprengel, Michael

Sraml, Matjaz

Srečec, Siniša

Srndovic, Milan

Sroka, Wlodzimierz
Stachewich, Urszula

Staiano, Antonino

Stala, Robert

Stamatelatou, Aikaterini

Stamatelos, Anastasios

Stamatelos, Tassos

Stanciu, Camelia

Stanciu, Dorin

Staniec, Kamil

Stanislav, Pejovic

Stansby, Peter K

Starace, Giuseppe

Stasińska, Beata

Staszak, Katarzyna

Stathopoulos, Panagiotis

Staub, Sebastian

Stavrakakis, George S.

Stavroulakis, Georgios E.

Stawarz, Marcin

Stefanescu, Razvan

Steinberger-Wilckens, Robert

Steinmann, Wolf-Dieter

Steller, Janusz

Stelson, Kim

Stensen, Jan Age

Stephen, Bruce

Stephens, Thomas

Stephenson, Max O.

Stępień, Sławomir

Stetter, Ralf

Stevanovic, Ivica

Stevenson, Bradley

Stiebler, Manfred

Stievano, Lorenzo

Stifter, Matthias

Stluka, Petr

Stoevesandt, Bernhard

Stokes, Christopher

Stolf, Patricia

Stoppato, Anna

Stork, Milan

Stoyanov, Borislav

Strantzali, Eleni

Strasser, Thomas

Straub, Jeremy

Strauss, Alfred

Streckiene, Giedre

Streimikiene, Dalia

Strezov, Vladimir

Strielkowski, Wadim

Stritih, Uros

Strizhak, Pavel A.

Struzik, Michal

Strzemiecka, Beata

Stull, Roland

Štumberger, Gorazd

Stuparu, Adrian

Stylianakis, Minas 
$\mathrm{Su}$, Chia-Hung

$\mathrm{Su}$, Heng-Yi

$\mathrm{Su}$, Ming-Shou

Su, Qinglin

$\mathrm{Su}$, Ruoyu

Su, Yuehong

Suárez, Rafael

Subbiah, Jegadesan

Subburaj, Anitha Sarah

Subiantoro, Alison

Subramaniam, Aravinth

Suciu, George

Sugar Gabor, Oliviu

Sugihara, Hideharu

Suh, Dong Hee

Suh, In-Soo

Suh, Jangwon

Suh, Nam P.

Suk, Sunhee

Sullivan, Pierre

Sułowicz, Maciej

Sum, Amadeu

Sumereder, Christof

Sun, Cheng-Gong

Sun, Guanghao

Sun, Rui

Sun, Yongxia

Sun, Zeyi

Sunderland, Peter

Sundqvist, Jan-Olov

Sung, Andrew

Sung, Jaeyong

Suslov, Sergey

Suszanowicz, Dariusz

Suwarno, Suwarno

Suzuki, Yasushi

Svecko, Rajko

Svendsen, Svend

Svensson, Bo $\mathrm{H}$.

Svetlík, Jozef

Sweeney, Daniel

Świt, Grzegorz

Syed, Amer

Szabó, László

Szabo, Lorand

Szala, Mirosław

Szalwinski, Chris

Szczegielniak, Tomasz

Szcześniak, Paweł

Szekely, Gyorgy

Szeląg, Adam

Szeląg, Maciej

Szeląg, Wojciech

Szeleszczuk, Lukasz

Szewczyk, Roman

Szombara, Stanisław

Szoplik, Jolanta

Szóstak, Mariusz
Szulc, Justyna

Szyszlak-Bargłowicz, Joanna

Tadanaga, Kiyoharu

Taebi, Amirtahà

Taeihagh, Araz

Taherdangkoo, Reza

Taherzadeh, Mohammad

Tahmasebi, Pejman

Tajaddodianfar, Farid

Tajalli, Armin

Tajiri, Kazuya

Takano, Toshiyuki

Takata, Kazutaka

Takeya, Satoshi

Takosoglu, Jakub Emanuel

Takyi, Gabriel

Talaat, Ahmed

Tam, Ivan CK

Tamer, Aykut

Tammi, Kari

Tamminen, Tarja

Tamošaitienè, Jolanta

Tamosiunas, Andrius

Tampio, Elina

Tan, Alex Yong Kwang

Tan, Chee-Keong

Tan, Kuang-Hsiung

Tanabe, Yuji

Tanaka, Yugo

Tang, Wenbo

Tang, Xu

Tao, Shanwen

Tapoglou, Nikolaos

Tarabieh, Khaled

Tarczewski, Tomasz

Targiel, Krzysztof

Tariq, Mohammad

Tarroja, Brian

Tartakovsky, Leonid

Tauveron, Nicolas

Tavares Ribeiro, Jorge Manuel

Taylor, Peter

Tchanche, Bertrand Fankam

Tcvetkov, Pavel

Tedeschi, Elisabetta

Tedesco, Francesco

Teghil, Roberto

Tehrani, Nima H.

Teixido-Figueras, Jordi

Tejedor-Flores, Nathalia

Teleszewski, Tomasz

Teli, Despoina

Temiz, Irina

Tenorio, Victor Octavio

Tenreiro, Claudio

Teodoriu, Catalin

Teprovich, Joseph

Terry, Stephen D. 
Teschke, Markus

Teslya, Anna

Tessarolo, Alberto

Teuvo, Suntio

Tevetkov, Nikolai

Thaduri, Adithya

Tham, Kwok Wai

Thang, Duong Quang

Theocharis, John B.

Theodosiou, Theodoros

Theotokatos, Gerasimos

Thiagarajan, Divya

Thiebot, Jérôme

Thirugnanam, Kannan

Thompson, Gregory J.

Thomsen, Jonas Bjerg

Thorarinsdottir, Ragnheidur

Tian, Wei

Tian, Yuan

Tinti, Francesco

Tirler, Werner

Tiron-Tudor, Adriana

Tiseira, Andrés Omar

Tkach, Alexander

Tochkov, Kiril

Toczylowska-Maminska, Renata

Todde, Giuseppe

Todeschini, Grazia

Toelle, Brian

Toffolo, Andrea

Togashi, Eisuke

Togneri, Michael

Tokuhiro, Akira

Toma, Lucian

Tomac, Ingrid

Toman, Petr

Tomasz, Falborski

Tomoiaga, Bogdan

Tomoyoshi, Nishiumura

Tong, Zheming

Toniolo, Horacio

Tonoli, Andrea

Topić, Danijel

Töreyin, Hakan

Torgal, F.Pacheco

Tornese, Fabiana

Torok, Adam

Torre, Salvo

Torres, José

Torresi, Marco

Torres-Moreno, Jose Luis

Torres-Sospedra, Joaquín

Torrijos, Iván Darío Piñerez

Torti, Emanuele

Toshifumi, Ise

Tóth, Tünde

Tourlidakis, Antonios

Toussaint, Udo Von
Tran, Khanh-Quang

Tran, Thanh Toan

Trevizoli, Paulo Vinicius

Tribioli, Laura

Trimboli, Scott

Trivedi, Rahul

Triviño, Alicia

Trnka, Pavel

Trojanek, Radosław

Tronchin, Lamberto

Trusso Sfrazzetto, Giuseppe

Trzmiel, Grzegorz

Tsai, Ang-Chen

Tsai, Huan-Liang

Tsai, Mi-Ching

Tsai, Ming-Tang

Tsai, Sang-Bing

Tsai, Wen-Chang

Tsai, Wen-Hsien

Tsamos, Konstantinos

Tsangrassoulis, Aris

Tsekouras, G.J.

Tseng, Ming-Lang

Tseng, Shu-Ming

Tseng, Victor Farm-Guoo

Tsiakas, Ilias

Tsikaloudaki, Katerina

Tsimpanogiannis, Ioannis N.

Tsinos, Christos

Tsionas, Mike

Tsiropoulou, Eirini Eleni

Tsitouras, Charalampos

Tso, Chi Yan

Tsolas, Ioannis E.

Tsoutsos, Theocharis

Tsow, Francis (Tsing)

Tsuji, Yutaka

Tsvetkov, Nikolai

Tsvetkov, Pavel

Tsyganova, Julia V

Tsyshevskiy, Roman

$\mathrm{Tu}$, Jih-Fu

Tu, Zhengkai

Tucciarelli, Tullio

Tudisca, Salvatore

Tudoroiu, Nicolae

Tuljak Suban, Danijela

Tulu, Ihsan

Tungal, Richa

Tupènaitè, Laura

Turco, Emilio

Tureczek, Alexander

Turki, Faical

Turki, Sadok

Turkoglu, Kamran

Tutak, Magdalena

Tutak, Wojciech

Tutuncu, Azra 
Tveit, Tor-Martin

Twaha, Ssennoga

Tynjala, Tero

Tytła, Malwina

Tziourtzioumis, Dimitrios

Ubong, Etim

Uchida, Susumu

Uemichi, Akane

Uezu, Kazuya

Ugarte, Sergio

Ugrina, Marin

Uhlig, Tobias

Ukrainczyk, Neven

Ulazia, Alain

Ullah, AMM Sharif

Ulloa, Carlos

Uno, Masatoshi

Unterrieder, Christoph

Unterweger, Christoph

Upare, Pravin P.

Urban, Petr

Urbina, Antonio

Urness, Kimberly

Uruba, Václav

Usai, Alessia

Usaola, Julio

Usher, Tedi-Marie

Ushida, Akiomi

Uslar, Mathias

Uteau, Daniel

Utgikar, Vivek

Utili, Stefano

Vadahanambi, Sridhar

Vadiee, Amir

Vafiadis, Kyriakos

Vagapov, Yuriy

Vagiona, Dimitra

Vagnoli, Stefano

Vahedi, Hani

Vaiana, Rosolino

Vakkilainen, Esa

Valavanides, Marios S.

Valdiserri, Paolo

Vale, Brenda

Valera Martinez, Diego Luis

Valerio Iungo, Giacomo

Valero, Carme

Valero-Lara, Pedro

Valero-Verdú, Sergio

Vallati, Andrea

Vallianatos, Filippos

Valogianni, Konstantina

Valouch, Viktor

Valvano, Stefano

Van Den Bossche, Alex

Van Der Blij, Nils H.

Van der Heijden, Rob

Van Der Waerden, Peter
Van Dijk, Meine Pieter

Van Loenen, Evert J.

Van Neste, Charles

Van Rooijen, Willem Frederik Geert

Van Vliet, Oscar

Van Wee, Bert

Vanbeveren, Stefan

Vandevelde, Lieven

Vaněk, Michal

Vaninsky, Alexander

Vannucchi, Valentina

Vanti, Maurizio

Vanus, Jan

VanZwieten, James H.

Varfolomejeva, Renata

Varga, Pal

Varua, Maria Estela

Vasilatos, Charalampos

Vasile, Andrei Jean

Vasta, Salvatore

Vaunat, Jean

Vaz, Warren

Vázquez, Enrique Personal

Vázquez, Javier

Vedyagin, Aleksey A.

Veiga Rodrigues, Carlos

Vela, Francisco Gómez

Velasco, Leticia F.

Velasco-Vélez, Juan Jesús

Velázquez-Martí, Borja

Venditti, Mattia

Venegas, Javier Freire

Venegas, María

Veneri, Ottorino

Venkatesan, Ramachandran

Venturini, Mauro

Venugopal, Vengatesan

Vera García, Francisco

Verde, Paola

Verga, Francesca

Vergura, Silvano

Verticale, Giacomo

Vespignani, Joaquin L.

Vezzetti, Enrico

Viaro, Umberto

Viccione, Giacomo

Vicidomini, Maria

Vicinanza, Diego

Victor, Nadejda

Vidhi, Rachana

Vidmar, Peter

Vien, Quoc-Tuan

Vieru, Dumitru

Vigevano, L.

Viggiano, Annarita

Vignaroli, Gianluca

Vignati, Michele

Vigueras Rodríguez, Antonio 
Vilchis-Rodriguez, Damian

Villegas-Pico, Hugo Nestor

Vinagre, Eugénia

Vinayakumar, K B

Vinogradov, Sergey

Vinuesa, Ricardo

Viola, Fabio

Vita, Vasiliki

Vitale, Gianpaolo

Vitetta, Giorgio

Viva, Federico A.

Viveiros, Carla

Viviano, Antonino

Vivo, Paola

Vizcaíno, Arturo J.

Vižintin, Goran

Vizureanu, Petrică

Vizza, Pasquale

Vlachokostas, Alex

Vlachos, Dimitrios

Vlachos, Nicholas

Vlad, Ciprian

Vlad, Diaconita

Vlontzos, George

Vo, Minh

Vo, Truong

Vobecky, Jan

Volkov, Konstantin

Volosencu, Constantin

Von Zur Muehlen, Peter

Vora, Ankit

Vorechovsky, Miroslav

Vorobiev, Oleg

Vowinckel, Bernhard

Vrakas, Apostolos

Vranayova, Zuzana

Vrančić, Damir

$\mathrm{Vu}$, Tuyen $\mathrm{V}$.

Vujević, Slavko

Vukobratovic, Marko

Vulin, Domagoj

Vulpe, Alexandru

Vuong, Quan-Hoang

Vyskočil, Vlastimil

Wachowski, Marcin

Wachs, Israel

Wadrzyk, Mariusz

Wagner, Andreas

Wahiduzzaman, MD

Waindok, Andrzej

Walczak, Krzysztof

Walker, Adam

Walker, Sean

Walker, Stuart

Walsh, Wilfred

Walters, George

Walther, Thomas

Wan, Gang
Wang, Aining

Wang, Baomin

Wang, Bin

Wang, Can

Wang, Chen-Hao

Wang, Danling

Wang, Dong

Wang, Fu-Cheng

Wang, Guochao

Wang, Hao

Wang, Hsiang-Chen

Wang, Hui

Wang, Jidong

Wang, Jihong

Wang, Jinsheng

Wang, Kai

Wang, Kun

Wang, Liguo

Wang, Lingling

Wang, Liwei

Wang, Ming-Shyan

Wang, Mu-chun

Wang, Ning

Wang, Pei Jen

Wang, Qing

Wang, Qinyi

Wang, Ruofan

Wang, Shiyan

Wang, Shu

Wang, Shuoqin

Wang, Taiping

Wang, Xia

Wang, Xidong

Wang, Xin

Wang, Xizu

Wang, Yan

Wang, Yang

Wang, Yeqi

Wang, Yong

Wang, Yubo

Wang, Yun

Wang, Zhan

Wang, Zhenyu

Wang, Zhichao

Wang, Zhongwei

Wanjiku, John

Wanniarachchi, Ayal

Ward, John

Ward, Phil

Wardach, Marcin

Wardman, John

Warren, Clive

Waschl, Harald

Wase, Nishikant

Washko, Frank M.

Watanabe, Koichi

Watanabe, Tsunemi

Wątróbski, Jarosław 
Watson, Francesca

Waxman, Michael

Weaver, Wayne W.

Webber, Beau

Weber, Felix

Weber, Ludger

Webley, Paul

Wegener, Christoph

Węgrzyński, Wojciech

Wei, Chih-Chiang

Wei, Chun

Wei, Kaya

Wei, Yang

Wei, Zhongbao

Weidlich, Ingo

Weinberger, Christian

Weindl, Christian

Weinstein, Lee A.

Weiß, Andreas

Weiss, Leland

Weitz, Keith

Welgamage Don, Aakash

Welkenhuysen, Kris

Wen, Baole

Wen, Chih-Yu

Werner, Sven

Wernik, Jacek

Weron, Rafal

Westaway, Robert

Wetterlund, Elisabeth

White, Martin

Wibowo, David

Wickramasinghe, Chathurika

Widger, Phillip

Widiatmojo, Arif

Widl, Edmund

Wieland, Christoph

Wieleba, Wojciech

Wierzbicki, Mirosław

Wiese, Jürgen

Wilburn, Kathleen M.

Wild, Graham

Wilhelm, Jay

Williams, Alan

Williamson, Geoffrey A.

Wilson, David Gordon

Windom, Bret

Wishart, Jeffrey

Wisniewski, Adam

Wisniewski, Remigiusz

Witos, Miroslaw

Witz, Christian

Włodarczyk, Paweł P.

Wodak, Josh

Wojciechowski, Adam

Wojciechowski, Łukasz

Wojciechowski, Rafal M.

Wojcik, Janusz
Wojdyga, Krzysztof

Wojnicki, Igor

Woldemariam, Daniel

Wolszczak, Piotr

Wong, Ka-Chun

Wong, Wing-Keung

Wood, David

Wouters, P.A.A.F.

Wright, Mark Mba

$\mathrm{Wu}$, Bisheng

$\mathrm{Wu}, \mathrm{Di}$

$\mathrm{Wu}$, Donghai

$\mathrm{Wu}$, Huixuan

$\mathrm{Wu}$, Jinn-Chang

$\mathrm{Wu}, \mathrm{Kan}$

Wu, Keliu

$\mathrm{Wu}$, Longfei

$\mathrm{Wu}$, Shenghua

$\mathrm{Wu}$, Shih-Jeh

Wu, Ta Yeong

Wu, Tzi-Yi

$\mathrm{Wu}$, Wei

$\mathrm{Wu}, \mathrm{We}-\mathrm{Tao}$

$\mathrm{Wu}$, Xuejian

$\mathrm{Wu}$, Yuan-Kang

$\mathrm{Wu}, \mathrm{Yu}-\mathrm{Chi}$

Wu, Yu-En

$\mathrm{Wu}$, Yuh-Yih

$\mathrm{Wu}, \mathrm{Yu}$-Ting

$\mathrm{Wu}$, Zhongze

Wu, Ziping

Wycisk, Ryszard

Wydra, Michal

Wysocki, Marian

Xavier, Antonio

Xia, Bing

Xia, Guanglin

Xia, Yidong

Xiao, Boqi

Xiao, Ting

Xie, Fei

Xie, Jing

Xie, Jingrui (Rain)

Xie, Kunpeng

Xie, $\mathrm{Pu}$

Xie, Quan

Xie, Zhijian

$\mathrm{Xu}$, Guang

$\mathrm{Xu}$, Guanshui

Xu, Jiagang

$X \mathrm{u}, \mathrm{Ke}$

$\mathrm{Xu}, \mathrm{Li}$

$\mathrm{Xu}, \mathrm{Lu}$

Xu, Shanshan

$\mathrm{Xu}$, Wanli

$\mathrm{Xu}$, Weixing

$\mathrm{Xu}, \mathrm{Yao}$

$\mathrm{Xu}$, Yuhao 


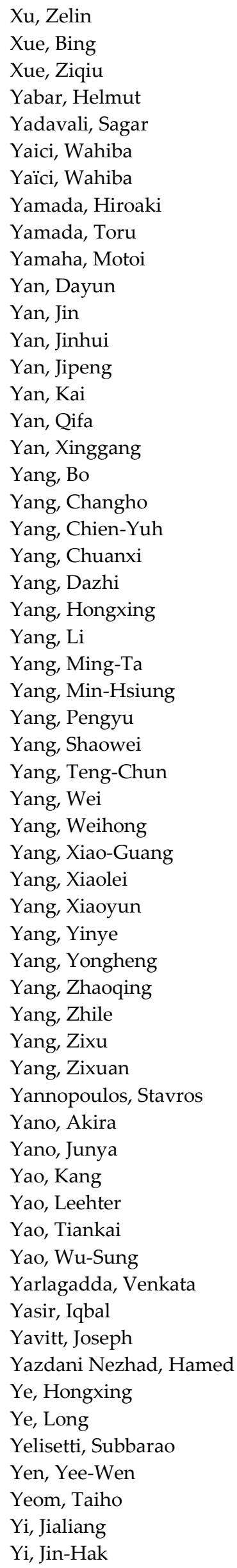

Yildiz, Özgür

Yilmaz, Erol

Yilmaz, Muhittin

Yin, Bangsheng

Yin, Fanghui

Yin, Huiming

Yin, Shunde

Yin, Wuliang

Yogeesh, Maruthi

Yoo, Junsoo

Yoon, Jeoung Seok

Yoon, Sam-Ki

Yoon, Sung-Guk

Yordanov, Georgi Hristov

Yoshida, Shigeo

Yoshino, Hiroshi

Yoshino, Toshihiko

Younes Seyedi, Younes

Younis, Adnan

Yousefi-Talouki, Arzhang

Yousif, Jabar H.

Youssef, Tarek

Yoza, Brandon A.

Yperman, Jan

Yu, Chi-Yang

$\mathrm{Yu}, \mathrm{Fu}-\mathrm{Wing}$

$\mathrm{Yu}, \mathrm{Hao}$

Yu, Hsin Her

Yu, Huilong

$\mathrm{Yu}$, Jaecheul

$\mathrm{Yu}$, Samson

$\mathrm{Yu}$, Shengwei

$\mathrm{Yu}$, Shih-Heng

$\mathrm{Yu}$, Simon

Yu, Tzyy Leon

Yu, Xun

$\mathrm{Yu}$, Yang

Yu, Yi-Hsiang

Yuan, Jihui

Yuan, Min-Hao

Yuan, Qingwang

Yuen, Anthony Chun Yin

Yuen, Chau

Yum, Jun Ho

Yun, Geun Young

Yun, Rin

Yun, Tae Sup

Yunusa-Kaltungo, Akilu

Yunusov, Timur

Yurchenko, Olena

Zabkowski, Tomasz

Zacariah, Hildenbrand

Zachariades, Christos

Zacharof, Myrto

Zaghari, Bahareh

Zając, Grzegorz

Zajc, Matej

Zajec, Peter 
Zakinyan, Arthur

Zamani, Nematollah

Zamboni, Giorgio

Zannis, Theodoros

Zannotti, Marco

Zanotti Fragonara, Luca

Zapata, Jaime Wladimir

Zarnikau, Jay

Zarzuelo, Carmen

Zavadskas, Edmundas Kazimieras

Zavos, Anastasios

Zazpe, Raul

Zbeeb, Khaled

Zbořil, František

Zdankus, Tadas

Zdenek Muller, Zdenek

Zea Escamilla, Edwin

Zeghmati, Belkacem

Zehetbauer, Paul

Zeiler, Wim

Zemite, Laila

Zemke, Julian

Zenebe Degefa, Merkebu

Zeng, Jianwu

Zenger, Kai

Zetterling, Carl-Mikael

Zeyghami, Mehdi

Zhan, Junpeng

Zhan, Lingwei

Zhang, Bo

Zhang, Chen

Zhang, Cheng

Zhang, Daiqiang

Zhang, Fan

Zhang, Haijun

Zhang, Hongliang

Zhang, Jianhua

Zhang, Jie

Zhang, Junyi

Zhang, Kuangyuan

Zhang, Lin

Zhang, Meng

Zhang, Rongpeng

Zhang, Ruiyong

Zhang, Shuaifang

Zhang, Tieling

Zhang, Wei

Zhang, Wennan

Zhang, Xian-Ming

Zhang, Xiantao

Zhang, Xiaogang

Zhang, Xiaping

Zhang, Xingxing

Zhang, Xuefeng

Zhang, Xueqing

Zhang, Xuewei

Zhang, Xuyang

Zhang, Yan
Zhang, Yao

Zhang, Yifeng

Zhang, Yiming

Zhang, Yu

Zhang, Yudong

Zhang, Yuna

Zhang, Zhe

Zhang, Zhien

Zhang, Ziang

Zhao, Bote

Zhao, Evan Wenbo

Zhao, Hangbo

Zhao, Linhui

Zhao, Qiuying

Zhao, Wei

Zhao, Xianbo

Zhao, Xudong

Zhao, Yapu

Zhao, Zeyu

Zhao, Zhenghang

Zheng, Bo

Zheng, Sixu

Zheng, Yi

Zheng, Zhaoliang

Zhou, Dao

Zhou, Jiafeng

Zhou, Joe

Zhou, Peng

Zhou, Qian

Zhou, Ran

Zhou, Xuezhe

Zhou, You

Zhou, Zhengping

Zhou, Zhongfu

Zhu, Dibin

Zhu, Huayang

Zhu, Jiadeng

Zhu, Jianjun

Zhu, Jie

Zhu, Mingming

Zhu, Shan

Zhu, Xiaowei

Zhuang, Jun

Zhuang, Luwen

Zhuang, Shiqiang

Zhuang, Xiaoying

Zi, Yunlong

Zidan, Aboelsood

Ziemba, Paweł

Zigan, Lars

Zikria, Yousaf Bin

Zilinskas, Antanas

Zima, Piotr

Zimmerman, William B.

Zimon, Dominik

Ziogou, Chrysovalantou

Zisser, Wolfhard

Zissis, George 
Zizzo, Gaetano

Zobaa, Ahmed

Zou, Changfu

Zou, Shangyan

Zou, Yu

Zubia, Itziar

Zubieta, Luis

Zulueta, Ekaitz
Zumel, Pablo

Zunino, Pietro

Zuo, Lihua

Zuppa, Leonardo Acho

Zwarycz-Makles, Katarzyna

Zygmanowski, Marcin

Żyromski, Andrzej

(c) 2019 by the author. Licensee MDPI, Basel, Switzerland. This article is an open access article distributed under the terms and conditions of the Creative Commons Attribution (CC BY) license (http://creativecommons.org/licenses/by/4.0/). 